\title{
Cyclic Generalized Vectors for Algebras of Unbounded Operators
}

\author{
By \\ Atsushi INOUE * and Witold KARwOwSKI **
}

\section{$\S 1$. Introduction}

Algebras of unbounded operators called $O^{*}$-algebras appear in rather broad pure mathematical context (operator theory, topological *-algebras, representations of Lie algebras etc.) and the physical applications (the Wightman quantum field theory, unbounded CCR-algebras, quantum groups etc.). This motivated systematic studies carried on for about three decades.

One of the most serious difficulties in the investigations of $O^{*}$-algebras is caused by the pathological relations between the invariant subspaces and projections of the commutant $\mathscr{M}_{\text {w }}^{\prime}$ of an $O^{*}$-algebra $\mathscr{M}[6,15\rfloor$ which prevents $\mathscr{M}$ from being spatially isomorphic with a direct sum of $O^{*}$-algebras possessing cyclic vectors in general. In other words only a very special subfamily of $O^{*}$-algebras have representations with cyclic vectors. On the other hand the concept of cyclic vector proved to be very useful for studies of $O^{*}$-algebras $[4,9,10,12,19]$. These facts suggest that perhaps a generalization of the notion of cyclic vector would provide a useful tool for investigations of a wider class of $O^{*}$-algebras. In this note we shall pursue this idea.

To be more specific, let $\mathscr{M}$ be a closed $O^{*}$-algebra on a dense subspace $\mathscr{D}$ in a Hibert space $\mathscr{H}$ satisfying $\mathscr{M}_{\mathrm{w}}^{\prime} \mathscr{D} \subset \mathscr{D}$. The motivations as well as the guidelines for the choice of appropriate definition of generalized vector emerge from following three problems :

(i) Vectors in $\mathscr{H}-\mathscr{D}$. It seems reasonable to require that any vector $\xi \in \mathscr{H}$ should be a generalized vector for $\mathscr{M}$. Observe that if $\xi \in \mathscr{D}$ then it defines a linear map $\lambda_{\xi}$ from $\mathscr{M}$ into $\mathscr{D}$ by

$$
\lambda_{\xi}(A)=A \xi, \quad A \in \mathscr{M}
$$

Communicated by H. Araki, October 26, 1992. Revised August 27, 1993.

1991 Mathematics Subject Classifications : 47D40

* Department of Applied Mathematics, Fukuoka University, Fukuoka, Japan.

** Institute of Theoretical Physics, University of Wroclaw, 50205 Wroclaw, ul. Cybulskiego 36, Poland. 
This map has following property

$$
\lambda_{\xi}(X A)=X \lambda_{\xi}(A) \text { for all } X, A \in \mathscr{M} \text {. }
$$

This suggests that if $\xi \in \mathscr{H}-\mathscr{D}$ then it can still define a linear map from a (possibly trivial) subspace $\mathscr{D}\left(\lambda_{\xi}\right) \subset \mathscr{M}$ into $\mathscr{D}$. If we wish to preserve the above multiplicative relation, then $\mathscr{D}\left(\lambda_{\xi}\right)$ has to be a left ideal of $\mathscr{M}$. This requirements result (see Sect. $3)$ in the following :

For any $\xi \in \mathscr{H}, \lambda_{\xi}$ is a linear map defined by

$$
\begin{aligned}
& D\left(\lambda_{\xi}\right)=\left\{X \in \mathscr{M} ; \xi \in \mathscr{D}\left(X^{\dagger *}\right) \text { and } X^{\dagger *} \xi \in \mathscr{D}\right\} \\
& \lambda_{\xi}(X)=X^{\dagger *} \xi, \quad X \in \mathscr{D}\left(\lambda_{\xi}\right) .
\end{aligned}
$$

(ii) Weights on $O^{*}$-algebras : It is well known that weights play an important role for the study of the structure of von Neumann algebras $[2,5,21]$, and so it seems useful to extend the notion of weights on von Neumann algebras to $O^{*}$-algebras. A map $\varphi$ of $\mathscr{P}(\mathscr{M}) \equiv\left\{\sum_{k=1}^{n} X_{k}^{\dagger} X_{k} ; X_{k} \in \mathscr{M}(k=1,2, \ldots, n), n \in \mathbb{N}\right\}$ into $[0,+\infty]$ is said to be a weight if $\varphi(\boldsymbol{A}+\boldsymbol{B})=\varphi(\boldsymbol{A})+\varphi(\boldsymbol{B})$ and $\varphi(\lambda \boldsymbol{A})=\lambda \varphi(\boldsymbol{A})$ for $A, B \in \mathscr{P}(\mathscr{M})$ and $\lambda \geq 0$. Then the GNS-construction $\left(\pi_{\varphi}, \lambda_{\varphi}, \mathscr{H}_{\varphi}\right)$ is well-defined.

Taking $\lambda_{\xi}$ in (i) and $\lambda_{\varphi}$ in (ii) into consideration, we define the notion of generalized vectors for $\mathscr{M}$ as follows : A map $\lambda$ of $\mathscr{M}$ into $\mathscr{D}$ is said to be a generalized vector for $\mathscr{M}$ if it is a linear map of a left ideal $D(\lambda)$ of $\mathscr{M}$ into $\mathscr{D}$ satisfying $\lambda(X A)=X \lambda(A)$ for all $X \in \mathscr{M}$ and $A \in D(\lambda)$. A generalized vector $\lambda$ for $\mathscr{M}$ is said to be cyclic if $\lambda(D(\lambda))$ is dense in $\mathscr{H}$.

(iii) A generalization of the Tomita-Takesaki theory to $O^{*}$-algebras : TomitaTakesaki theory plays an important role for a study of structures of von Neumann algebras and for a study of quantum physics, and so it is desirable to extend the results of Tomita-Takesaki theory to $O^{*}$-algebras. With this viewpoint, in the previous paper [9] one of us A.I. defined and studied the notions of standard system and modular system which made it possible to develop the Tomita-Takesaki theory in $O^{*}$-algebras with cyclic vector. In this paper we shall extend the concepts of standard systems and modular systems to $O^{*}$-algebras with cyclic generalized vector by the following procedure :

Let $(\mathscr{M}, \lambda)$ be a pair of a closed $O^{*}$-algebra $\mathscr{M}$ on $\mathscr{D}$ in $\mathscr{H}$ satisfying $\mathscr{M}_{\mathrm{w}}^{\prime} \mathscr{D} \subset \mathscr{D}$ and a generalized vector $\lambda$ for $\mathscr{M}$ satisfying $\lambda\left(\left(D(\lambda) \cap D(\lambda)^{\dagger}\right)^{2}\right)$ is total in $\mathscr{H}$. Then we define three commutants $\lambda^{\prime}, \lambda^{\sigma}$ and $\lambda^{c}$ and the bicommutants $\lambda^{\prime \prime}, \lambda^{\sigma \sigma}, \lambda^{c c}$ of $\lambda$ which are generalized vectors for the von Neumann algebras $\mathscr{M}_{\mathrm{w}}^{\prime}$ and $\left(\mathscr{M}_{\mathrm{w}}^{\prime}\right)^{\prime}$, respectively. Suppose $\lambda^{\prime}\left(\left(D\left(\lambda^{\prime}\right) \cap D\left(\lambda^{\prime}\right)^{*}\right)^{2}\right)$ is total in $\mathscr{H}$. Then, the map $\lambda(X) \rightarrow \lambda\left(X^{\dagger}\right), \quad X \in D(\lambda) \cap D(\lambda)^{\dagger}$ and $\lambda^{\prime \prime}(A) \rightarrow \lambda^{\prime \prime}\left(A^{*}\right), A \in D\left(\lambda^{\prime \prime}\right) \cap D\left(\lambda^{\prime \prime}\right) *$ are closable in $\mathscr{H}$ and their closures are denoted by $S_{\lambda}$ and $S_{\lambda^{\prime \prime}}$, respectively. Let $S_{\lambda}=$ $J_{\lambda} \Delta_{\lambda^{\prime \prime}}^{1 / 2}$ and $S_{\lambda^{\prime \prime}}=J_{\lambda^{\prime \prime}} \Delta_{\lambda^{\prime \prime}}^{1 / 2}$ be the polar decompositions of $S_{\lambda}$ and $S_{\lambda^{\prime \prime}}$, respectively. 
Then we see that $S_{\lambda} \subset S_{\lambda^{\prime \prime}}$, and $J_{\lambda^{\prime \prime}}\left(\mathscr{M}_{\mathrm{w}}^{\prime}\right)^{\prime} J_{\lambda^{\prime \prime}}=\mathscr{M}_{\mathrm{w}}^{\prime}$ and $\Delta_{\lambda^{\prime \prime}}^{i t}\left(\mathscr{M}_{\mathrm{w}}^{\prime}\right)^{\prime} \Delta_{\lambda^{\prime \prime}}^{-i t}=\left(\mathscr{M}_{\mathrm{w}}^{\prime}\right)^{\prime}$ for all $t \in \mathbf{R}$ by the Tomita fundamental theorem. But, we don't know how the unitary group $\left\{\Delta_{\lambda^{\prime \prime}}^{i t}\right\}_{t \in \mathbf{R}}$ acts on the $O^{*}$-algebra $\mathscr{M}$, and so we define a system which in this respect has the best properties: $\left(\mathscr{M}, \lambda, \lambda^{\prime}\right)$ is said to be a standard system if

$$
\begin{aligned}
& \Delta_{\lambda^{\prime \prime}}^{i t} \mathscr{D}=\mathscr{D} \text { and } \Delta_{\lambda^{\prime \prime}}^{i t} \mathscr{M} \Delta_{\lambda^{\prime \prime}}^{-i t}=\mathscr{M}(t \in \mathbf{R}), \\
& \Delta_{\lambda^{\prime \prime}}^{i t}\left(D(\lambda) \cap D(\lambda)^{\dagger}\right) \Delta_{\lambda^{\prime \prime}}^{-i t}=D(\lambda) \cap D(\lambda)^{\dagger}(t \in \mathbf{R}) .
\end{aligned}
$$

Then we shall show that if $\left(\mathscr{M}, \lambda, \lambda^{\prime}\right)$ is a standard system, then $S_{\lambda}=S_{\lambda^{\prime \prime}}, \quad\left\{\sigma_{t}^{\lambda}\right\}_{t \in \mathbf{R}}$ $\left(\sigma_{t}^{\lambda}(X) \equiv \Delta_{\lambda}^{i t} X \Delta_{\lambda}^{-i t}, X \in \mathscr{M}, t \in \mathbf{R}\right)$ is a one-parameter group of $*$-automorphisms of $\mathscr{M}$ and $\lambda$ satisfies the KMS-condition with respect to $\left\{\sigma_{t}^{\lambda}\right\}_{t \in \mathbf{R}}$. Suppose $\lambda^{c}\left(\left(D\left(\lambda^{c}\right)\right.\right.$ $\left.\cap D\left(\lambda^{c}\right)^{\dagger}\right)^{2}$ ) is total in $\mathscr{H}$. Then $S_{\lambda} c c, J_{\lambda} c c$ and $\Delta_{\lambda^{c c}}$ are similarly defined. $\left(\mathscr{M}, \lambda, \lambda^{c}\right)$ is said to be an essentially standard system if

(S3) $\Delta_{\lambda c c}^{i t} \mathscr{D}=\mathscr{D}$ and $\Delta_{\lambda^{c c}}^{i t} \mathscr{M} \Delta_{\lambda^{c c}}^{-i t}=\mathscr{M}(t \in \mathbf{R})$,

and further if

$$
\Delta_{\lambda^{c c}}^{i t}\left(\mathrm{D}(\lambda) \cap \mathrm{D}(\lambda)^{\dagger}\right) \Delta_{\lambda^{c c}}^{-i t}=\mathrm{D}(\lambda) \cap \mathrm{D}(\lambda)^{\dagger}(\mathrm{t} \in \mathbf{R})
$$

then $\left(\mathscr{M}, \lambda, \lambda^{c}\right)$ is said to be a standard system. We shall show that if $\left(\mathscr{M}, \lambda, \lambda^{c}\right)$ is a standard system, then $\left(\mathscr{M}, \lambda, \lambda^{\prime}\right)$ is a standard system and $S_{\lambda}=S_{\lambda^{\prime \prime}}=S_{\lambda^{c c}}$. Furthermore, we shall show that if $\left(\mathscr{M}, \lambda, \lambda^{c}\right)$ is an essentially standard system, then there exists a cyclic generalized vector $\lambda_{e}$ for $\mathscr{M}$ which is an extension of $\lambda$ such that $\left(\mathscr{M}, \lambda_{e}, \lambda_{e}^{c}\right)$ is a standard system. By relaxing the requirements (S3) and (S4), we define the notion of modular systems which enable us to develop unbounded Tomita-Takesaki theory and which is more applicable to examples. Roughly speaking, $\left(\mathscr{M}, \lambda, \lambda^{c}\right)$ is a modular system if there exists a good subdomain in $\mathscr{D}$ which is $\mathscr{M}$-invariant and $\left\{\Delta_{\lambda}^{t c} c c\right\}$-invariant. We shall show that if $\Gamma=\left(\mathscr{M}, \lambda, \lambda^{c}\right)$ is a modular system, then there exists a standard system $\left(\mathscr{U}(\Gamma), \lambda_{s}, \lambda_{s}^{c}\right)$ which is an extension of $\left(\mathscr{M}, \lambda, \lambda^{c}\right)$ in a certain sense. We shall finally apply these results to $\left(\mathscr{M}, \lambda_{\xi_{0}}, \lambda_{\xi_{0}}^{c}\right)$, where $\xi_{0}$ is a cyclic and separating vector for $\left(\mathscr{M}_{\mathrm{w}}^{\prime}\right)^{\prime}$, and give standard systems and moduler systems for unbounded CCR-algebras.

In this paper we have treated only $O^{*}$-algebras $\mathscr{M}$ on $\mathscr{D}$ satisfying $\mathscr{M}_{\mathrm{w}}^{\prime} \mathscr{D} \subset \mathscr{D}$. In more general case, that is when $\mathscr{M}_{\mathrm{w}}^{\prime}$ is not a von Neumann algebra but there exists a good von Neumann algebra $\mathscr{B}$ such that $\mathscr{B}^{\prime} \subset \mathscr{M}_{\text {w }}^{\prime}$, we can consider cyclic and separating systems, standard systems and modular systems using the induced extension $\iota_{\mathscr{B}}(\mathscr{M})$ of $\mathscr{M}$ by $\mathscr{B}^{\prime}[11,19]$. But, in order to clear the arguments we have assumed $\mathscr{M}_{\mathrm{w}}^{\prime} \mathscr{D} \subset \mathscr{D}$ in this paper (in this case, $\mathscr{M}_{\mathrm{w}}^{\prime}$ is a von Neumann algebra and $\mathscr{B}^{\prime}=\mathscr{M}_{\mathrm{w}}^{\prime}$ ).

In a forthcoming paper we shall investigate when a general $O^{*}$-algebra is 
algebraically (or spatially) isomorphic to an $O^{*}$-algebra with a cyclic generalized vector, and the problem of constructing standard (or modular systems) from standard von Neumann algebras. Furthermore, we shall study weights on $O^{*}$-algebras in detail.

\section{§ 2. Preliminaries}

In this section we state some definitions and basic properties concerning $O^{*}$-algebras $[3,6,11,13,15,18,19]$.

Let $\mathscr{D}$ be a dense subspace in a Hilbert space $\mathscr{H}$. We denote by $\mathscr{L}^{\dagger}(\mathscr{D})$ the set of all linear operators $X$ from $\mathscr{D}$ into $\mathscr{D}$ such that $\mathscr{D}\left(X^{*}\right) \supset \mathscr{D}$ and $X^{*} \mathscr{D} \subset \mathscr{D}$. Then $\mathscr{L}^{\dagger}(\mathscr{D})$ is a $*$-algebra with the usual operations and the involution $X \rightarrow X^{\dagger} \equiv X^{*}\lceil\mathscr{D}$. A *-subalgebra of $\mathscr{L}^{\dagger}(\mathscr{D})$ is called by Schmüdgen [19] an $O^{*}$-algebra on $\mathscr{D}$ in $\mathscr{H}$ but other authors often write $O_{p}^{*}$-algebra. Throughout this paper we assume that an $O^{*}$-algebra has always an identity operator. Let $\mathscr{M}$ be an $O^{*}$-algebra on $\mathscr{D}$. A locally convex topology on $\mathscr{D}$ defined by a family $\left\{\|\|_{X} ; X \in \mathscr{M}\right\}$ of the seminorms : $\|\xi\|_{X}=\|X \xi\|(\xi \in \mathscr{D})$ is called the induced topology on $\mathscr{D}$, and denoted by $t_{\mathscr{M}}$. If the locally convex space $\left(\mathscr{D}, t_{\mathscr{M}}\right)$ is complete, then $\mathscr{M}$ is said to be closed. We put

$$
\tilde{\mathscr{D}}(\mathscr{M})=\bigcap_{\boldsymbol{X} \in \mathscr{M}} \mathscr{D}(\bar{X}) \text { and } \tilde{X}=\bar{X}\lceil\tilde{\mathscr{D}}(\mathscr{M})(X \in \mathscr{M}) .
$$

Then $\tilde{\mathscr{D}}(\mathscr{M})$ is identical with the completion of $\left(\mathscr{D}, t_{\mathscr{M}}\right)$ and $\tilde{\mathscr{M}} \equiv\{\tilde{X} ; X \in \mathscr{M}\}$ is a closed $O^{*}$-algebra on $\tilde{D}(\mathscr{M})$ which is the smallest closed extension of $\mathscr{M}$ and it is called the closure of $\mathscr{M}$. Hence $\mathscr{M}$ is called closed if and only if $\mathscr{D}=\tilde{\mathscr{D}}(\mathscr{M})$. A vector $\xi_{0}$ of $\mathscr{D}$ is said to be cyclic (resp. strongly cyclic) for $\mathscr{M}$ if $\mathscr{M} \xi_{0}$ is dense in the Hilbert space $\mathscr{H}$ (resp. the locally convex space $\left.\left(\mathscr{D}, t_{\mathscr{M}}\right)\right)$. If $\mathscr{D}^{*}(\mathscr{M}) \equiv \bigcap_{x \in \mathscr{H}} \mathscr{D}\left(X^{*}\right)$ $=\tilde{\mathscr{D}}(\mathscr{M})$, then $\mathscr{M}$ is said to be essentially self-adjoint, and if $\mathscr{D}^{*}(\mathscr{M})=\mathscr{D}$, then $\mathscr{M}$ is said to be self-adjoint. Let $m$ be an $\mathscr{M}$-invariant subspace of $\mathscr{D}$ and $\mathscr{M}\lceil m$ the set of all restrictions $X\left\lceil m\right.$ of $X \in \mathscr{M}$ to $m$. Then $\mathscr{M}\left\lceil m\right.$ is an $O^{*}$-algebra on $m$. If $\mathscr{M}\lceil m$ is essentially self-adjoint, then $\mathbb{E}_{m} \mathscr{D}$ equals the closure $\bar{m}^{t} \mathscr{M}$ of $m$ with respect to the induced topology $t_{\mathscr{M}}$, where $E_{m} \equiv$ Proj. $\bar{m}$. Conversely if $\mathscr{M}$ is self-adjoint and $E_{m} \mathscr{D}$ $=\bar{m}^{t} \mathscr{M}$, then $\mathscr{M}\lceil m$ is essentially self-adjoint [15]. An element $\xi$ of $\mathscr{D}$ is said to be a self-adjoint vector for $\mathscr{M}$ if $\mathscr{M}\lceil\mathscr{M} \xi$ is essentially self-adjoint [6].

We define the weak commutant $\mathscr{M}_{\mathrm{w}}^{t}$ of a $\dagger$-invariant subset $\mathscr{M}$ of $\mathscr{L}^{\dagger}(\mathscr{D})$ as follows :

$$
\mathscr{M}_{\mathrm{w}}^{\prime}=\left\{C \in \mathscr{B}(\mathscr{H}) ;(C X \xi \mid \eta)=\left(C \xi \mid X^{\dagger} \eta\right) \text { for all } \xi, \eta \in \mathscr{D} \text { and } X \in \mathscr{M}\right\},
$$

where $\mathscr{B}(\mathscr{H})$ is the set of all bounded linear operators on $\mathscr{H}$. Then $\mathscr{M}_{\mathrm{w}}^{\prime}$ is a $*$ invariant weakly closed subspace of $\mathscr{B}(\mathscr{H})$, but it is not necessarily an algebra. It has been known that if $\mathscr{M}$ is a self-adjoint $O^{*}$-algebra, then $\mathscr{M}_{\text {w }}^{\prime} \subset \mathscr{D}$; and further 
$\mathscr{M}_{\mathrm{w}}^{\prime} \mathscr{D} \subset \mathscr{D}$ if and only if $\mathscr{M}_{\mathrm{w}}^{\prime}$ is a von Neumann algebra and $\bar{X}$ is affiliated with $\left(\mathscr{M}_{\mathrm{w}}^{\prime}\right)^{\prime}$ for each $X \in \mathscr{M}[15,19]$.

We fix the notations which will be used in this paper. Let $\mathscr{A}$ be a $*$-algebra. For subsets $m, n$ of $\mathscr{A}$ we define subsets $m^{*}, m n$ and $m^{2}$ of $\mathscr{A}$ by

$$
\begin{aligned}
& m^{*}=\left\{x^{*} ; x \in m\right\}, \\
& m n=\{x y ; x \in m, y \in n\}, \\
& m^{2} \equiv m m=\{x y ; x, y \in m\} .
\end{aligned}
$$

When $\mathscr{A}$ is a $*$-algebra without identity, we denote by $\mathscr{A}_{1}$ the $*$-algebra obtained from $\mathscr{A}$ by adjoining an identity.

\section{§3. Cyclic Generalized Vectors}

In this section we define the notion of cyclic generalized vector for an $O^{*}$-algebra which is a generalization of that of cyclic vector. Let $\mathscr{M}$ be an $O^{*}$-algebra on a dense subspace $\mathscr{D}$ in a Hilbert space $\mathscr{H}$.

Definition 3. 1. A map $\lambda$ of $\mathscr{M}$ into $\mathscr{D}$ is said to be a generalized vector for $\mathscr{M}$ if the following conditions hold:

(i) The domain $D(\lambda)$ of $\lambda$ is a left ideal of $\mathscr{M}$.

(ii) $\lambda$ is a linear map of $D(\lambda)$ into $\mathscr{D}$.

(iii) $\lambda(X A)=X \lambda(A)$ for all $X \in \mathscr{M}$ and $A \in D(\lambda)$.

$A$ generalized vector $\lambda$ for $\mathscr{M}$ is said to be cyclic (resp. strongly cyclic) if $\lambda(D(\lambda)$ ) is dense in $\mathscr{H}\left(\right.$ resp. $\left.\left(\mathscr{D}, t_{\mathscr{M}}\right)\right)$.

Let $\lambda$ be a generalized vector for $\mathscr{M}$. Then, the closure of an $O^{*}$-algebra $\{X\lceil\lambda(D(\lambda)) ; X \in \mathscr{M}\}$ on $\lambda(D(\lambda))$ in $\mathscr{H}(\lambda) \equiv \overline{\lambda(D(\lambda))}$ is denoted by $\mathscr{M}(\lambda)$ and called the $O^{*}$-algebra generated by $\lambda . \mathscr{M}(\lambda)$ is a restriction of $\mathscr{M}$. Even if $\lambda$ is cyclic, $\mathscr{M}(\lambda)$ does not necessarily equal $\mathscr{M}$ (see [19], Example 8.3.18). If however $\lambda$ is strongly cyclic, then $\mathscr{M}(\lambda)=\mathscr{M}$. Let $\lambda_{1}$ and $\lambda_{2}$ be generalized vectors for $\mathscr{M}$. If $D\left(\lambda_{1}\right) \subset D\left(\lambda_{2}\right)$ and $\lambda_{1}(X)=\lambda_{2}(X)$ for each $X \in D\left(\lambda_{1}\right)$, then $\lambda_{2}$ is said to be an extension of $\lambda_{1}$ and we write $\lambda_{2} \supset \lambda_{1}$.

We give some examples of generalized vectors for $O^{*}$-algebras.

Example 3. 2. (i) Let $\mathscr{M}$ be an $O^{*}$-algebra on $\mathscr{D}$ in $\mathscr{H}$ and $\xi \in \mathscr{H}$. We put

$$
\left\{\begin{array}{l}
D\left(\lambda_{\xi}\right)=\left\{X \in \mathscr{M} ; \xi \in \mathscr{D}\left(X^{\dagger *}\right) \text { and } X^{\dagger *} \xi \in \mathscr{D}\right\}, \\
\lambda_{\xi}(X)=X^{\dagger *} \xi, X \in D\left(\lambda_{\xi}\right) .
\end{array}\right.
$$

Then $\lambda_{\xi}$ is a generalized vector for $\mathscr{M}$. It is clear that $\lambda_{\xi}$ is cyclic (resp. strongly 
cyclic) if and only if $\left\{X^{\dagger *} \xi ; X \in D\left(\lambda_{\xi}\right)\right\}$ is dense in $\mathscr{H}\left(\right.$ resp. $\left.\left(\mathscr{D}, t_{\mathscr{M}}\right)\right)$. We remark that putting

$$
\left\{\begin{array}{l}
D(\lambda)=\{X \in \mathscr{M} ; \xi \in \mathscr{D}(\bar{X}) \text { and } \bar{X} \xi \in \mathscr{D}\} \\
\lambda(X)=\bar{X} \xi, X \in D(\lambda)
\end{array}\right.
$$

$D(\lambda)$ which is not necessarily a left ideal of $\mathscr{M}$ because $\overline{X+Y} \not \supset \bar{X}+\bar{Y}$ in general, and so $\lambda$ is not a generalized vector for $\mathscr{M}$.

(ii) Let $\mathscr{A}$ be a $*$-algebra and $f$ a positive linear functional on $\mathscr{A}$. Let $\left(\pi_{f}\right.$, $\left.\lambda_{f}, \mathscr{H}_{f}\right)$ be the GNS-construction for $f$. If $1 \in \mathscr{A}$, then $\lambda_{f}(1)$ is a strongly cyclic vector for $\pi_{f}(\mathscr{A})$. Let $1 \notin \notin \mathscr{A}$. Suppose $\pi_{f}(\boldsymbol{x}) \rightarrow \lambda_{f}(\boldsymbol{x})$ is a map, that is, $\pi_{f}(\boldsymbol{x})=0$ implies $\lambda_{f}(x)=0$, and then put

$$
\lambda\left(\pi_{f}(x)\right)=\lambda_{f}(x), x \in \mathscr{A} .
$$

Then $\lambda$ is a strongly cyclic generalized vector for the $O^{*}$-algebra $\pi_{f}(\mathscr{A})_{1}$ with $D(\lambda)=\pi_{f}(\mathscr{A})$.

(iii) Let $\mathscr{A}$ be a $*$-algebra and let

$$
\mathscr{P}(\mathscr{A})=\left\{\sum_{k=1}^{n} x_{k}^{*} x_{k} ; x_{k} \in \mathscr{A}(k=1,2, \ldots, n), n \in \mathbb{N}\right\} .
$$

A map $\varphi$ of $\mathscr{P}(\mathscr{A})$ into $\mathbb{R}^{+} \cup\{+\infty\}$ is said to be a weight on $\mathscr{P}(\mathscr{A})$ if

(i) $\varphi\left(\alpha x^{*} x\right)=\alpha \varphi\left(x^{*} x\right), x \in \mathscr{A}, \alpha \geq 0$;

(ii) $\varphi(a+b)=\varphi(a)+\varphi(b), a, b \in \mathscr{P}(\mathscr{A})$, where $0 \cdot(+\infty)=0$.

Let $\varphi$ be a weight on $\mathscr{P}(\mathscr{A})$. We put

$$
\begin{aligned}
n_{\varphi}^{0} & =\left\{a \in \mathscr{A} ; \varphi\left(a^{*} a\right)<\infty\right\}, \\
m_{\varphi} & =\text { linear span of }\left\{a^{*} a ; a \in n_{\varphi}^{0}\right\}, \\
\dot{\varphi}\left(\sum_{k} \alpha_{k} a_{k}^{*} a_{k}\right) & =\sum_{k} \alpha_{k} \varphi\left(a_{k}^{*} a_{k}\right) \text { for } \sum_{k} \alpha_{k} a_{k}^{*} a_{k} \in m_{\varphi} .
\end{aligned}
$$

Since $\left(a_{1}+a_{2}\right) *\left(a_{1}+a_{2}\right)+\left(a_{1}-a_{2}\right) *\left(a_{1}-a_{2}\right)=2\left(a_{1}^{*} a_{1}+a_{2}^{*} a_{2}\right)\left(a_{1}, a_{2} \in n_{\varphi}^{0}\right)$, it follows that $n_{\varphi}^{0}$ is a subspace of $\mathscr{A}$ and $\dot{\varphi}$ is a well-defined linear functional on $m_{\varphi}$. But, $n_{\varphi}^{0}$ is not necessarily a left ideal of $\mathscr{A}$, and so we put

$$
n_{\varphi}=\left\{a \in n_{\varphi}^{0} ; x a \in n_{\varphi}^{0} \text { for all } x \in \mathscr{A}\right\}
$$

It is easily shown that $n_{\varphi}$ is a left ideal of $\mathscr{A}$ and

$$
\left|\dot{\varphi}\left(b^{*} a\right)\right|^{2} \leq \varphi\left(b^{*} b\right) \varphi\left(a^{*} a\right) \text { for all } a, b \in n_{\varphi} \text {. }
$$

We put

$$
N_{\varphi}=\left\{a \in n_{\varphi} ; \varphi\left(a^{*} a\right)=0\right\},
$$




$$
\lambda_{\varphi}(a)=a+N_{\varphi} \in n_{\varphi} / N_{\varphi}, a \in n_{\varphi} .
$$

Then $\lambda_{\varphi}\left(n_{\varphi}\right) \equiv n_{\varphi} / N_{\varphi}$ is a pre-Hilbert space with the inner product

$$
\left(\lambda_{\varphi}(a) \mid \lambda_{\varphi}(b)\right)=\dot{\varphi}\left(b^{*} a\right), a, b \in n_{\varphi} .
$$

We denote by $\mathscr{H}_{\varphi}$ the Hilbert space obtained by the completion of the pre-Hilbert space $\lambda_{\varphi}\left(n_{\varphi}\right)$. We define a $*$-representation $\pi_{\varphi}^{0}$ of $\mathscr{A}$ by

$$
\pi_{\varphi}^{0}(x) \lambda_{\varphi}(a)=\lambda_{\varphi}(x a), x \in \mathscr{A}, a \in n_{\varphi},
$$

and denote by $\pi_{\varphi}$ the closure of $\pi_{\varphi}^{0}$. We call the triple $\left(\pi_{\varphi}, \lambda_{\varphi}, \mathscr{H}_{\varphi}\right)$ the GNS-construction for $\varphi$. Suppose $\pi_{\varphi}(a) \rightarrow \lambda_{\varphi}(a)\left(a \in n_{\varphi}\right)$ is a map and then put

$$
\lambda\left(\pi_{\varphi}(a)\right)=\lambda_{\varphi}(a), a \in n_{\varphi} .
$$

Then $\lambda$ is a strongly cyclic generalized vector for $\pi_{\varphi}(\mathscr{A})_{1}$ with the domain $\pi_{\varphi}\left(n_{\varphi}\right)$.

\section{§ 4. Cyclic and Separating Systems}

In this section we define commutants and bicommutants of cyclic generalized vectors for $O^{*}$-algebras and then study cyclic and separating systems. Throughout this section let $(\mathscr{M}, \lambda)$ be a pair of a closed $O^{*}$-algebra $\mathscr{M}$ on $\mathscr{D}$ in $\mathscr{H}$ and a generalized vector $\lambda$ for $\mathscr{M}$ satisfying

(i) $\mathscr{M}_{\mathrm{w}}^{\prime} \mathscr{D} \subset \mathscr{D}$,

(ii) $\lambda\left(D(\lambda)^{\dagger} D(\lambda)\right)$ is total in $\mathscr{H}$.

We first define three commutants of $\lambda$ as follows :

$$
\begin{aligned}
& \begin{cases}D\left(\lambda^{\prime}\right)=\left\{K \in \mathscr{M}_{\mathrm{w}}^{\prime} ;\right. & { }^{\exists} \xi_{K} \in \bigcap_{X \in D(\lambda)} \mathscr{D}(\bar{X}) \text { s.t. } \\
& \left.K \lambda(X)=\bar{X} \xi_{K} \text { for all } X \in D(\lambda)\right\}, \\
\lambda^{\prime}(K)=\xi_{K}, K \in D\left(\lambda^{\prime}\right) .\end{cases} \\
& \begin{cases}D\left(\lambda^{\sigma}\right)=\left\{K \in \mathscr{M}_{\mathrm{w}}^{\prime} ;\right. & { }^{\exists} \xi_{K} \in \bigcap_{X \in D(\lambda)} \mathscr{D}\left(X^{\dagger *}\right) \text { s.t. } \\
& \left.K \lambda(X)=X^{\dagger *} \xi_{K} \text { for all } X \in D(\lambda)\right\}, \\
\lambda^{\sigma}(K)=\xi_{K}, K \in D\left(\lambda^{\sigma}\right) .\end{cases} \\
& \left\{\begin{array}{cc}
D\left(\lambda^{c}\right)=\left\{K \in \mathscr{M}_{\mathrm{w}}^{\prime} ;\right. & { }^{\exists} \xi_{K} \in \mathscr{D} \text { s.t. } \\
\lambda^{c}(K)=\xi_{K}, & K \in D(X)=X\left(\lambda^{c}\right) .
\end{array}\right.
\end{aligned}
$$

Then we have the following 
Proposition 4. 1. $\lambda^{\prime}, \lambda^{\sigma}$ and $\lambda^{c}$ are generalized vectors for the von Neumann algebra $\mathscr{M}_{w}^{\prime}$ and $\lambda^{c} \subset \lambda^{\prime} \subset \lambda^{\circ}$.

Proof. Since $\lambda\left(D(\lambda)^{\dagger} D(\lambda)\right)$ is total in $\mathscr{H}$, it follows that $\xi_{K}$ is uniquely determined for $K \in D\left(\lambda^{\prime}\right), D\left(\lambda^{\prime}\right)$ is a subspace of $\mathscr{M}_{\mathrm{w}}^{\prime}$ and $\lambda^{\prime}$ is a linear map of $D\left(\lambda^{\prime}\right)$ into $\bigcap_{x \in D(\lambda)} \mathscr{D}(\bar{X})$. Take arbitrary $C \in \mathscr{M}_{\mathrm{w}}^{\prime}$ and $K \in D\left(\lambda^{\prime}\right)$. Then, since $\bar{X}$ is affiliated with $\left(\mathscr{M}_{\mathrm{w}}^{\prime}\right)^{\prime}$ for each $X \in D(\lambda)$ (by (i)), it follows that $C \lambda^{\prime}(\mathbb{K}) \in$ $\bigcap_{X \in D(\lambda)} \mathscr{D}(\bar{X})$ and $C K \lambda(X)=C \bar{X} \lambda^{\prime}(K)=\bar{X} C \lambda^{\prime}(K)$ for all $X \in D(\lambda)$. Hence we have

$$
C K \in D\left(\lambda^{\prime}\right) \text { and } \lambda^{\prime}(C K)=C \lambda^{\prime}(K)
$$

for all $C \in \mathscr{M}_{\mathrm{w}}^{\prime}$ and $K \in D\left(\lambda^{\prime}\right)$. Therefore, $D\left(\lambda^{\prime}\right)$ is a left ideal of $\mathscr{M}_{\mathrm{w}}^{\prime}$ and $\lambda^{\prime}$ is a generalized vector for $\mathscr{M}_{\mathrm{w}}^{\prime}$. We can similarly show that $\lambda^{\sigma}$ and $\lambda^{c}$ are generalized vectors for $\mathscr{M}_{\mathrm{w}}^{\prime}$. It is clear that $\lambda^{c} \subset \lambda^{\prime} \subset \lambda^{\sigma}$.

Definition 4. 2. $\left(\mathscr{M}, \lambda, \lambda^{\prime}\right)\left(\right.$ resp. $\left.\left(\mathscr{M}, \lambda, \lambda^{\sigma}\right),\left(\mathscr{M}, \lambda, \lambda^{c}\right)\right)$ is said to be a cyclic and separating system if it satisfies the conditions (i), (ii) and moreover

(iii) $\lambda^{\prime}\left(D\left(\lambda^{\prime}\right) * D\left(\lambda^{\prime}\right)\right)\left(\right.$ resp. $\left.\lambda^{\sigma}\left(D\left(\lambda^{\sigma}\right) * D\left(\lambda^{\sigma}\right)\right), \lambda^{c}\left(D\left(\lambda^{c}\right)^{*} D\left(\lambda^{c}\right)\right)\right)$ is total in $\mathscr{H}$.

In case $\mathscr{M}$ consists of bounded operators we have $\lambda^{\prime}=\lambda^{\sigma}=\lambda^{c}$, and so we simply call $(\mathscr{M}, \lambda)$ a cyclic and separating system provided $\left(\mathscr{M}, \lambda, \lambda^{\prime}\right)$ is cyclic and separating.

Let $\left(\mathscr{M}, \lambda, \lambda^{\prime}\right)$ be a cyclic and separating system. Then $\lambda^{\prime}$ is a cyclic generalized vector for the von Neumann algebra $\mathscr{M}_{\mathrm{w}}^{\prime}$ satisfying $\lambda^{\prime}\left(D\left(\lambda^{\prime}\right)^{*} D\left(\lambda^{\prime}\right)\right)$ is total in $\mathscr{H}$, and so by Proposition 4. 1 three commutants $\left(\lambda^{\prime}\right)^{\prime},\left(\lambda^{\prime}\right)^{c}$ and $\left(\lambda^{\prime}\right)^{o}$ of $\lambda^{\prime}$ are well-defined and they are identical. To emphasize the commutant of $\lambda^{\prime}$ we use the notation $\left(\lambda^{\prime}\right)^{\prime}$ (simply, $\lambda^{\prime \prime}$ ) as the commutant of $\lambda^{\prime}$, and then $\lambda^{\prime \prime}$ is defined as follows :

$$
\begin{cases}D\left(\lambda^{\prime \prime}\right)=\left\{A \in\left(\mathscr{M}_{\mathrm{w}}^{\prime}\right)^{\prime} ;\right. & { }^{\exists} \xi_{A} \in \mathscr{H} \text { s.t. } \\ & \left.A \lambda^{\prime}(K)=K \xi_{A} \text { for all } K \in D\left(\lambda^{\prime}\right)\right\}, \\ \lambda^{\prime \prime}(A)=\xi_{A}, A \in D\left(\lambda^{\prime \prime}\right) . & \end{cases}
$$

It is easily shown that $\lambda^{\prime \prime}$ is well-defined and it is a linear map of the subspace $D\left(\lambda^{\prime \prime}\right)$ of $\left(\mathscr{M}_{\mathrm{w}}^{\prime}\right)^{\prime}$ into $\mathscr{H}$.

Proposition 4.3. Suppose $\left(\mathscr{M}, \lambda, \lambda^{\prime}\right)$ is a cyclic and separating system. Then $\left(\left(\mathscr{M}_{\mathrm{w}}^{\prime}\right)^{\prime}, \lambda^{\prime \prime}\right)$ is a cyclic and separating system satisfying $\lambda^{\prime \prime \prime} \equiv\left(\lambda^{\prime \prime}\right)^{\prime}=\lambda^{\prime}$.

Proof. It is easily shown that $\lambda^{\prime \prime}$ is a generalized vector for $\left(\mathscr{M}_{\mathrm{w}}^{\prime}\right)^{\prime}$. We show $\lambda^{\prime \prime}\left(D\left(\lambda^{\prime \prime}\right)\right)$ is dense in $\mathscr{H}$. Take an arbitrary $X \in D(\lambda)$. Let $\bar{X}=U|\bar{X}|$ be the 
polar decomposition of $\bar{X}$ and $|\bar{X}|=\int_{0}^{\infty} t d E(t)$ the spectral resolutions of $|\bar{X}|$. We put

$$
E_{n}=\int_{0}^{n} d E(t) \text { and } X_{n}=\bar{X} E_{n}, n \in \mathbf{N}
$$

Since $\bar{X}$ is affiliated with $\left(\mathscr{M}_{\mathrm{w}}^{\prime}\right)^{\prime}$, it follows that $U, X_{n} \in\left(\mathscr{M}_{\mathrm{w}}^{\prime}\right)^{\prime}$ and

$$
\begin{aligned}
X_{n} \lambda^{\prime}(K)=U|\bar{X}| E_{n} \lambda^{\prime}(K) & =U E_{n} U^{*} \bar{X} \lambda^{\prime}(K) \\
& =U E_{n} U^{*} K \lambda(X) \\
& =K U E_{n} U^{*} \lambda(X)
\end{aligned}
$$

for all $K \in D\left(\lambda^{\prime}\right)$ and $n \in \mathbb{N}$. Hence we have

$$
X_{n} \in D\left(\lambda^{\prime \prime}\right) \text { and } \lambda^{\prime \prime}\left(X_{n}\right)=U E_{n} U^{*} \lambda(X), n \in \mathbb{N}
$$

Furthermore, since

$$
\begin{aligned}
\left(U U^{*} \lambda(\boldsymbol{X}) \mid \lambda^{\prime}\left(K_{1}^{*} K_{2}\right)\right) & =\left(U U^{*} K_{1} \lambda(X) \mid \lambda^{\prime}\left(K_{2}\right)\right) \\
& =\left(U U^{*} \bar{X} \lambda^{\prime}\left(K_{1}\right) \mid \lambda^{\prime}\left(K_{2}\right)\right) \\
& =\left(\bar{X} \lambda^{\prime}\left(K_{1}\right) \mid \lambda^{\prime}\left(K_{2}\right)\right) \\
& =\left(\lambda(X) \mid \lambda^{\prime}\left(K_{1}^{*} K_{2}\right)\right)
\end{aligned}
$$

for all $K_{1}, K_{2} \in D\left(\lambda^{\prime}\right)$ and $\lambda^{\prime}\left(D\left(\lambda^{\prime}\right) * D\left(\lambda^{\prime}\right)\right)$ is total in $\mathscr{H}$, it follows that

$$
U U^{*} \lambda(X)=\lambda(X)
$$

By (4.1) and (4.2) we have

$$
\lim _{n \rightarrow \infty} \lambda^{\prime \prime}\left(X_{n}\right)=\lambda(X)
$$

Furthermore, by the definition of $X_{n}$ we have

$$
\begin{aligned}
& \lim _{n \rightarrow \infty} X_{n} \xi=\bar{X} \xi, \quad \xi \in \mathscr{D}(\bar{X}), \\
& \lim _{n \rightarrow \infty} X_{n}^{*} \eta=X^{*} \eta, \quad \eta \in \mathscr{D}\left(X^{*}\right) .
\end{aligned}
$$

Since $\lambda(D(\lambda))$ is dense in $\mathscr{H}$, it follows from (4.1) and (4.3) that

$$
\lambda^{\prime \prime}\left(D\left(\lambda^{\prime \prime}\right)\right) \text { is dense in } \mathscr{H} \text {. }
$$


We next show that $\lambda^{\prime \prime}\left(D\left(\lambda^{\prime \prime}\right)^{*} D\left(\lambda^{\prime \prime}\right)\right)$ is total in $\mathscr{H}$. It follows from (4.1) and (4.5) that $\left\{A^{*} \lambda(Y) ; A \in D\left(\lambda^{\prime \prime}\right), Y \in D(\lambda)\right\}$ is dense for $\lambda\left(D(\lambda)^{\dagger} D((\lambda))\right.$. Furthermore, it follows from (4.6) that $\lambda^{\prime \prime}\left(D\left(\lambda^{\prime \prime}\right) * D\left(\lambda^{\prime \prime}\right)\right)$ is total in $\mathscr{H}$. Therefore, $\lambda^{\prime \prime \prime} \equiv\left(\lambda^{\prime \prime}\right)^{\prime}$ is well-defined by :

$$
\left\{\begin{array}{lr}
D\left(\lambda^{\prime \prime \prime}\right)=\left\{K \in \mathscr{M}_{\mathrm{w}}^{\prime} ;{ }^{\exists} \xi_{K} \in \mathscr{H}\right. & \text { s. t. } K \lambda^{\prime \prime}(A)=A \xi_{K} \\
\lambda^{\prime \prime \prime}(K)=\xi_{K}, & \text { for all } \left.A \in D\left(\lambda^{\prime \prime}\right)\right\},
\end{array}\right.
$$

It is clear that $\lambda^{\prime} \subset \lambda^{\prime \prime \prime}$. Hence, $\left(\left(\mathscr{M}_{\mathrm{w}}^{\prime}\right)^{\prime}, \lambda^{\prime \prime}\right)$ is a cyclic and separating system. We finally show $\lambda^{\prime \prime \prime}=\lambda^{\prime}$. Take arbitrary $K \in D\left(\lambda^{\prime \prime \prime}\right)$ and $X \in D(\lambda)$. Then it follows from (4.1) and (4.3) that

$$
\begin{aligned}
& \lim _{n \rightarrow \infty} E_{n} \lambda^{\prime \prime \prime}(K)=\lambda^{\prime \prime \prime}(K) \\
& \lim _{n \rightarrow \infty} \bar{X} E_{n} \lambda^{\prime \prime \prime}(K)=\lim _{n \rightarrow \infty} K \lambda^{\prime \prime}\left(X_{n}\right)=K \lambda(X)
\end{aligned}
$$

Therefore, $\lambda^{\prime \prime \prime}(K) \in \bigcap_{X \in D(\lambda)} \mathscr{D}(\bar{X})$ and $\bar{X} \lambda^{\prime \prime \prime}(K)=K \lambda(X)$ for all $X \in D(\lambda)$, and so $K \in$ $D\left(\lambda^{\prime}\right)$. This completes the proof.

Let $\left(\mathscr{M}, \lambda, \lambda^{\sigma}\right)$ be a cyclic and separating system. As defined $\lambda^{\prime \prime}$ we can define the commutant $\left(\lambda^{\sigma}\right)^{\sigma}$ (simply, $\left.\lambda^{\sigma \sigma}\right)$ as follows :

$$
\begin{cases}\left(\lambda^{\sigma \sigma}\right)=\left\{A \in\left(\mathscr{M}_{\mathrm{w}}^{\prime}\right)^{\prime} ;{ }^{\exists} \xi_{A} \in \mathscr{H}\right. & \text { s.t. } A \lambda^{\sigma}(K)=K \xi_{A} \\ \lambda^{\sigma \sigma}(A)=\xi_{A}, & \text { for all } \left.K \in D\left(\lambda^{\sigma}\right)\right\},\end{cases}
$$

Then we have the following

Proposition 4. 4. Suppose $\left(\mathscr{M}, \lambda, \lambda^{\sigma}\right)$ is a cyclic and separating system. Then $\left(\left(\mathscr{M}_{\mathrm{w}}^{\prime}\right)^{\prime}, \lambda^{\sigma \sigma}\right)$ is a cyclic and separating system satisfying $\lambda^{\sigma \sigma \sigma} \equiv\left(\lambda^{\sigma \sigma}\right)^{\sigma}=\lambda^{\sigma}$.

Proof. This is proved in similar to the proof of Proposition 4.3 considering the polar decomposition of $X^{\dagger *}$ and the spectral decomposition of $\left|X^{\dagger *}\right|, X \in D(\lambda)$.

Let $\left(\mathscr{M}, \lambda, \lambda^{c}\right)$ be a cyclic and separating system. As defined $\lambda^{\prime \prime}$ and $\lambda^{\sigma o}$ we can define the commutant $\left(\lambda^{c}\right)^{c}\left(\right.$ simply, $\left.\lambda^{c c}\right)$ as follows :

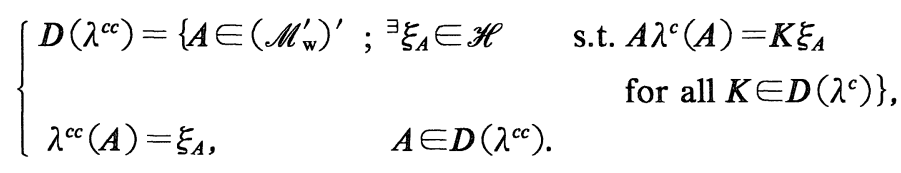


Then we have the following

Proposition 4. 5. Suppose $\left(\mathscr{M}, \lambda, \lambda^{c}\right)$ is a cyclic and separating system. Then $\left(\left(\mathscr{M}_{\mathrm{w}}^{\prime}\right)^{\prime}, \lambda^{c c}\right),\left(\left(\mathscr{M}_{\mathrm{w}}^{\prime}\right)^{\prime}, \lambda^{\prime \prime}\right)$ and $\left(\left(\mathscr{M}_{\mathrm{w}}^{\prime}\right)^{\prime}, \lambda^{\sigma \sigma}\right)$ are cyclic and separating systems satisfying $\lambda^{\sigma \sigma} \subset \lambda^{\prime \prime} \subset \lambda^{c c}$ and $\lambda^{\sigma} \supset \lambda^{\prime} \supset\left(\lambda^{c c}\right)^{c} \equiv \lambda^{c c c} \supset \lambda^{c}$.

Proof. It is clear that $\lambda^{c c}$ is a generalized vector for $\left(\mathscr{M}_{\mathrm{w}}^{\prime}\right)^{\prime}$. It follows from Proposition 4.1 that $\left(\mathscr{M}, \lambda, \lambda^{\prime}\right)$ and $\left(\mathscr{M}, \lambda, \lambda^{\sigma}\right)$ are cyclic and separating systems, which implies by Proposition $4.3,4.4$ that $\left(\left(\mathscr{M}_{\mathrm{w}}^{\prime}\right)^{\prime}, \lambda^{\prime \prime}\right)$ and $\left(\left(\mathscr{M}_{\mathrm{w}}^{\prime}\right)^{\prime}, \lambda^{\sigma \sigma}\right)$ are cyclic and separating systems. Further, since $\lambda^{\sigma \sigma} \subset \lambda^{\prime \prime} \subset \lambda^{c c}$, it follows that $\left(\left(\mathscr{M}_{\mathrm{w}}^{\prime}\right)^{\prime}\right.$, $\left.\lambda^{c c}\right)$ is a cyclic and separating system. By Proposition 4.1, 4.3 we have $\lambda^{c} \subset \lambda^{c c c} \subset \lambda^{\prime}$ $\subset \lambda^{\sigma}$.

We remark that $\lambda^{\prime \prime \prime}=\lambda^{\prime}$ and $\lambda^{\sigma \sigma \sigma}=\lambda^{\sigma}$, but $\lambda^{c c c} \neq \lambda^{c}$ in general as will be seen in Example 4.6, (6) below.

Example 4. 6. Let $\xi_{0} \in \mathscr{H}-\mathscr{D}$. Suppose $\xi_{0}$ is a cyclic and separating vector for $\left(\mathscr{M}_{\mathrm{w}}^{\prime}\right)^{\prime}$ and $\left\{X^{\dagger} Y^{\dagger *} \xi_{0} ; X, Y \in D\left(\lambda_{\xi_{0}}\right)\right\}$ is total in $\mathscr{H}$. Then we have the following results :

(1) $D\left(\lambda_{\xi_{0}}^{o}\right)=\mathscr{M}_{\mathrm{w}}^{\prime}$ and $\lambda_{\xi_{0}}^{\sigma}(C)=C \xi_{0}, C \in \mathscr{M}_{\mathrm{w}}^{\prime}$;

$$
D\left(\lambda_{\xi_{0}}^{\sigma o}\right)=\left(\mathscr{M}_{\mathrm{w}}^{\prime}\right)^{\prime} \text { and } \lambda_{\xi_{0}}^{\sigma o}(\boldsymbol{A})=\boldsymbol{A} \xi_{0}, A \in\left(\mathscr{M}_{\mathrm{w}}^{\prime}\right)^{\prime} .
$$

Hence $\left(\mathscr{M}, \lambda_{\xi_{0}}, \lambda_{\xi_{0}}^{g}\right)$ is a cyclic and separating system.

(2) $D\left(\lambda_{\xi_{0}}^{\prime}\right)=\left\{K \in \mathscr{M}_{\mathrm{w}}^{\prime} ; K \xi_{0} \in \underset{X \in D\left(\lambda_{\xi_{0}}\right)}{\bigcap} \mathscr{D}(\bar{X})\right\}$.

(3) $D\left(\lambda \xi_{\xi_{0}}^{c}\right)=\left\{K \in \mathscr{M}_{\mathrm{w}}^{\prime} ; K \xi_{0} \in \mathscr{D}\right\}$.

(4) $\lambda \lambda_{\xi_{0}}^{c} \subsetneq \lambda_{\xi_{0}}^{\prime} \subsetneq \lambda_{\xi_{0}}^{g}$ in general. In fact, if $\xi_{0} \in \bigcap_{X \in D\left(\lambda_{\xi_{0}}\right)} \mathscr{D}(\bar{X})$, then $\mathbf{I} \in D\left(\lambda_{\xi_{0}}^{\prime}\right)$ but

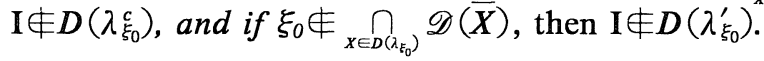

(5) The following statements are equivalent : $(\alpha)\left(\mathscr{M}, \lambda_{\xi_{0}}, \lambda_{\xi_{0}}^{\prime}\right)$ is a cyclic and separating system, $(\beta) \xi_{0} \in \bigcap_{X \in D\left(\lambda_{\xi_{0}}\right)} \mathscr{D}(\bar{X}),(\gamma) D\left(\lambda_{\xi_{0}}^{\prime}\right)=\mathscr{M}_{\mathrm{w}}^{\prime},(\delta) \lambda_{\xi_{0}}^{\prime}=\lambda_{\xi_{0}}^{g}$. In fact, suppose $\left(\mathscr{M}, \lambda_{\xi_{0}}, \lambda_{\xi_{0}}^{\prime}\right)$ is a cyclic and separating system. Then, since $\lambda_{\xi_{0}}^{\prime \prime} \supset \lambda_{\xi_{0}}^{\sigma o}$ and (1), we have $\lambda_{\xi_{0}}^{\prime \prime}=\lambda_{\xi_{0}}^{\sigma o}$, and so by Proposition 4.3, 4.4 $\lambda_{\xi_{0}}^{\prime}=\lambda_{\xi_{0}}^{\sigma}$. And then $D\left(\lambda_{\xi_{0}}^{\prime}\right)=$ $\mathscr{M}_{\mathrm{w}}^{\prime}$ by (1), and so $\xi_{0} \in \bigcap_{X \in D\left(\lambda_{E_{0}}\right)} \mathscr{D}(\bar{X})$ by (2). Conversely suppose $\xi_{0} \in \bigcap_{X \in D\left(\lambda_{\xi_{0}}\right)} \mathscr{D}(\bar{X})$. Then, $\mathrm{I} \in \boldsymbol{D}\left(\lambda_{\xi_{0}}^{\prime}\right)$, and so $\lambda_{\xi_{0}}=\lambda_{\xi_{0}}^{g}$. Therefore, $\left(\mathscr{M}, \lambda \xi_{\xi_{0}}, \lambda_{\xi_{0}}^{\prime}\right)$ is a cyclic and separating system.

(6) Suppose $\left(\mathscr{M}, \lambda_{\xi_{0}}, \lambda_{\xi_{0}}^{c}\right)$ is a cyclic and separating system. Then, $\lambda_{\xi_{0}}^{c c}=\lambda_{\xi_{0}}^{\prime \prime}=$ $\lambda_{\xi_{0}}^{\sigma \sigma}$ and $\lambda \lambda_{\xi_{0}}^{c} \subsetneq \lambda_{\xi_{0}}^{c c c}=\lambda_{\xi_{0}}^{\prime}=\lambda_{\xi_{0}}^{g}$.

In fact, since $D\left(\lambda^{c}\right) \cap D\left(\lambda^{c}\right) *$ is a nondegenerate *-subalgebra of $\mathscr{M}_{\mathrm{w}}^{\prime}$, there exists a net $\left\{K_{\alpha}\right\}$ in $D\left(\lambda^{c}\right) \cap D\left(\lambda^{c}\right)^{*}$ such that $0 \leq K_{\alpha} \leq l$ and $K_{\alpha} \uparrow l$ strongly. Then it follows that 


$$
\begin{gathered}
K_{\beta} C K_{\alpha} \in D\left(\lambda^{c}\right) \cap D\left(\lambda^{c}\right)^{*}, \\
\lim _{\alpha, \beta} \lambda \xi_{\xi_{0}}^{c}\left(K_{\beta} C K_{\alpha}\right)=K_{\beta} C K_{\alpha} \xi_{0} \longrightarrow C \xi_{0}=\lambda_{\xi_{0}}^{\prime}(C), \\
\lim _{\alpha, \beta} \lambda \xi_{\xi_{0}}^{c}\left(\left(K_{\beta} C K_{\alpha}\right)^{*}\right)=K_{\alpha} C^{*} K_{\beta} \xi_{0} \longrightarrow C^{*} \xi_{0}=\lambda_{\xi_{0}}^{\prime}\left(C^{*}\right)
\end{gathered}
$$

for each $C \in \mathscr{M}_{\mathrm{w}}^{\prime}$, which implies $\lambda_{\xi_{0}}^{c c}=\lambda_{\xi_{0}}^{\prime \prime}$. By (4), (5) we have $\lambda_{\xi_{0}}^{c c}=\lambda_{\xi_{0}}^{\prime \prime}=\lambda_{\xi_{0}}^{\sigma o}$ and $\lambda{ }_{\xi_{0}}^{c}$ $\subsetneq \lambda_{\xi_{0}}^{c c c}=\lambda_{\xi_{0}}^{\prime}=\lambda_{\xi_{0}}^{\sigma}$.

\section{§ 5. Standard Systems and Modular Systems}

In this section we study standard systems and modular systems which are able to develop the Tomita-Takesaki theory in $O^{*}$-algebras. Throughout this section let $(\mathscr{M}, \lambda)$ be a pair of a closed $O^{*}$-algebra $\mathscr{M}$ on a dense subspace $\mathscr{D}$ in a Hilbert space $\mathscr{H}$ and a generalized vector $\lambda$ for $\mathscr{M}$ satisfying

(i) $\mathscr{M}_{\mathrm{w}}^{\prime} \mathscr{D} \subset \mathscr{D}$;

(ii) $\lambda\left(\left(D(\lambda)^{\dagger} \cap D(\lambda)\right)^{2}\right)$ is total in $\mathscr{H}$.

By Proposition 4. 3 we have the following

Lemma 5. 1. Suppose

(iii) $\lambda^{\prime}\left(\left(D\left(\lambda^{\prime}\right) \cap D\left(\lambda^{\prime}\right)^{*}\right)^{2}\right)$ is total in $\mathscr{H}$.

Then the following statements hold :

(1) $\quad \lambda^{\prime}\left(D\left(\lambda^{\prime}\right) \cap D\left(\lambda^{\prime}\right)^{*}\right)$ is an achieved right Hilbert algebra in $\mathscr{H}$ equipped with the multiplication and the involution :

$$
\begin{aligned}
\lambda^{\prime}\left(K_{1}\right) \lambda^{\prime}\left(K_{2}\right)=\lambda^{\prime}\left(K_{2} K_{1}\right), & \lambda^{\prime}(\boldsymbol{K})^{b}=\lambda^{\prime}\left(K^{*}\right), \\
& K, K_{1}, K_{2} \in D\left(\lambda^{\prime}\right) \cap D\left(\lambda^{\prime}\right)^{*},
\end{aligned}
$$

and its right von Neumann algebra equals $\mathscr{M}_{\mathrm{w}}^{\prime}$.

(2) $\lambda^{\prime \prime}\left(D\left(\lambda^{\prime \prime}\right) \cap D\left(\lambda^{\prime \prime}\right)^{*}\right)$ is an achieved left Hilbert algebra in $\mathscr{H}$ equipped with the multiplication and the involution :

$$
\begin{array}{ll}
\lambda^{\prime \prime}(A) \lambda^{\prime \prime}(\mathbb{B})=\lambda^{\prime \prime}(A B), & \lambda^{\prime \prime}(\mathcal{A})^{\#}=\lambda^{\prime \prime}\left(\mathbb{A}^{*}\right), \\
& A, B \in D\left(\lambda^{\prime \prime}\right) \cap D\left(\lambda^{\prime \prime}\right)^{*},
\end{array}
$$

and it equals the commutant of the right Hilbert algebra $\lambda^{\prime}\left(D\left(\lambda^{\prime}\right) \cap D\left(\lambda^{\prime}\right)^{*}\right)$.

(3) $D\left(\lambda^{\prime \prime}\right)=\left\{A \in\left(\mathscr{M}_{\mathrm{w}}^{\prime}\right)^{\prime} ;{ }^{\exists} \xi_{A} \in \mathscr{H}\right.$ s.t. $A \lambda^{\prime}(K)=K \xi_{A}$ for all $K \in D\left(\lambda^{\prime}\right) \cap$ $\left.D\left(\lambda^{\prime}\right)^{*}\right\}$ and $\lambda^{\prime \prime}(A)=\xi_{A}$ for $A \in D\left(\lambda^{\prime \prime}\right)$.

(4) $D\left(\lambda^{\prime}\right)=D\left(\lambda^{\prime \prime \prime}\right)=\left\{K \in \mathscr{M}_{\mathrm{w}}^{\prime} ;{ }^{\exists} \xi_{K} \in \mathscr{H}\right.$ s.t. $K \lambda^{\prime \prime}(A)=A \xi_{K}$ for all $A \in \mathbb{D}\left(\lambda^{\prime \prime}\right)$ 
$\left.\cap D\left(\lambda^{\prime \prime}\right)^{*}\right\}$ and $\lambda^{\prime}(K)=\xi_{K}$ for $K \in D\left(\lambda^{\prime}\right)$.

Suppose $\lambda^{\prime}\left(\left(D\left(\lambda^{\prime}\right) \cap D\left(\lambda^{\prime}\right)^{*}\right)^{2}\right)$ is total in $\mathscr{H}$. By Lemma 5. 1 the map $\lambda^{\prime \prime}(A)$ $\rightarrow \lambda^{\prime \prime}\left(A^{*}\right), A \in D\left(\lambda^{\prime \prime}\right) \cap D\left(\lambda^{\prime \prime}\right) *$ is a closable conjugate-linear operator in $\mathscr{H}$, and so its closure is denoted by $S_{\lambda^{\prime \prime}}$. Since

$$
\left(\lambda(X) \mid \lambda^{\prime}\left(K_{1}^{*} K_{2}\right)\right)=\left(\lambda^{\prime}\left(K_{2}^{*} K_{1}\right) \mid \lambda\left(X^{\dagger}\right)\right)
$$

for all $X \in D(\lambda) \cap D(\lambda)^{\dagger}$ and $K_{1}, K_{2} \in D\left(\lambda^{\prime}\right) \cap D\left(\lambda^{\prime}\right)^{*}$, it follows that the map $\lambda(X) \rightarrow \lambda\left(X^{\dagger}\right), X \in D(\lambda) \cap D(\lambda)^{\dagger}$ is a closable conjugate-linear operator in $\mathscr{H}$, and so its closure is denoted by $S_{\lambda}$. Let $S_{\lambda^{\prime \prime}}=J_{\lambda^{\prime \prime}} \Delta_{\lambda^{\prime \prime}}^{1 / 2}$ and $S_{\lambda}=J_{\lambda} \Delta_{\lambda}^{1 / 2}$ be the polar decompositions of $S_{\lambda^{\prime \prime}}$ and $S_{\lambda}$, respectively.

Lemma 5. 2. Suppose $\lambda^{\prime}\left(\left(D\left(\lambda^{\prime}\right) \cap D\left(\lambda^{\prime}\right)^{*}\right)^{2}\right)$ is total in $\mathscr{H}$. Then $S_{\lambda} \subset S_{\lambda}^{\prime \prime}$.

Proof. Take an arbitrary $X \in D(\lambda) \cap D(\lambda)^{\dagger}$. Let $\bar{X}=V|\bar{X}|$ be the polar decomposition of $\bar{X},|\bar{X}|=\int_{0}^{\infty} t d E(t)$ the spectral resolution of $|\bar{X}|$ and $E_{n}=$ $\int_{0}^{n} d E(t)$ for $n \in \mathbf{N}$. We put

$$
X_{n}=\bar{X} E_{n}, n \in \mathbb{N}
$$

Then, by (4.1) and (4.2) we have

$$
X_{n} \in D\left(\lambda^{\prime \prime}\right), \lambda^{\prime \prime}\left(X_{n}\right)=U E_{n} U^{*} \lambda(X)(n \in \mathbb{N}) \text { and } U U^{*} \lambda(X)=\lambda(X)
$$

Furthermore, we have

$$
X_{n}^{*} \lambda^{\prime}(K)=E_{n} \overline{X^{\dagger}} \lambda^{\prime}(K)=K\left(E_{n} \lambda\left(X^{\dagger}\right)\right)
$$

for all $K \in D\left(\lambda^{\prime}\right)$. Hence we have

$$
X_{n}^{*} \in D\left(\lambda^{\prime \prime}\right) \text { and } \lambda^{\prime \prime}\left(X_{n}^{*}\right)=E_{n} \lambda\left(X^{\dagger}\right)(n \in \mathbb{N})
$$

By (5.1) and (5.2) we have

$$
X_{n} \in D\left(\lambda^{\prime \prime}\right) \cap D\left(\lambda^{\prime \prime}\right)^{*}, \lim _{n \rightarrow \infty} \lambda^{\prime \prime}\left(X_{n}\right)=\lambda(X) \text { and } \lim _{n \rightarrow \infty} \lambda^{\prime \prime}\left(X_{n}^{*}\right)=\lambda\left(X^{\dagger}\right)
$$

Therefore, $\lambda(X) \in \mathscr{D}\left(S_{\lambda^{\prime \prime}}\right)$ and $S_{\lambda^{\prime \prime}} \lambda(X)=\lambda\left(X^{\dagger}\right)=S_{\lambda} \lambda(X)$. This completes the proof. 
By the Tomita fundamental theorem [22] we have

$$
\begin{aligned}
& J_{\lambda^{\prime \prime}}\left(\mathscr{M}_{\mathrm{w}}^{\prime}\right)^{\prime} J_{\lambda^{\prime \prime}}=\mathscr{M}_{\mathrm{w}}^{\prime}, \\
& \sigma_{t}^{\lambda^{\prime \prime}}(A) \equiv \Delta_{\lambda^{\prime \prime}}^{i t} A \Delta_{\lambda^{\prime \prime}}^{-i t} \in\left(\mathscr{M}_{\mathrm{w}}^{\prime}\right)^{\prime} \quad\left(\mathrm{A} \in\left(\mathscr{M}_{\mathrm{w}}^{\prime}\right)^{\prime}, t \in \mathbf{R}\right), \\
& \sigma_{t}^{\lambda^{\prime \prime}}\left(D\left(\lambda^{\prime \prime}\right) \cap D\left(\lambda^{\prime \prime}\right)^{*}\right)=D\left(\lambda^{\prime \prime}\right) \cap D\left(\lambda^{\prime \prime}\right) * \text { and } \\
& \quad \lambda^{\prime \prime}\left(\sigma_{t}^{\lambda^{\prime \prime}}(B)\right)=\Delta_{\lambda^{\prime \prime}}^{i t} \lambda^{\prime \prime}(B)\left(B \in D\left(\lambda^{\prime \prime}\right) \cap D\left(\lambda^{\prime \prime}\right)^{*}, t \in \mathbf{R}\right), \\
& \sigma_{t}^{\lambda^{\prime \prime}}(C) \equiv \Delta_{\lambda^{\prime \prime}}^{i t} C \Delta_{\lambda^{\prime \prime}}^{-i t} \in \mathscr{M}_{\mathrm{w}}^{\prime} \quad\left(C \in \mathscr{M}_{\mathrm{w}}^{\prime}, t \in \mathbf{R}\right), \\
& \sigma_{t}^{\lambda^{\prime \prime}}\left(D\left(\lambda^{\prime}\right) \cap D\left(\lambda^{\prime}\right)^{*}\right)=D\left(\lambda^{\prime}\right) \cap D\left(\lambda^{\prime}\right)^{*} \text { and } \\
& \quad \lambda^{\prime}\left(\sigma_{t}^{\lambda^{\prime \prime}}(K)\right)=\Delta_{\lambda^{\prime \prime}}^{i t \prime} \lambda^{\prime}(K)\left(K \in D\left(\lambda^{\prime}\right) \cap D\left(\lambda^{\prime}\right)^{*}, t \in \mathbf{R}\right) .
\end{aligned}
$$

Furthermore, we have

$$
\begin{aligned}
& \sigma_{t}^{\lambda^{\prime \prime}}\left(D\left(\lambda^{\prime \prime}\right)\right)=D\left(\lambda^{\prime \prime}\right) \text { and } \lambda^{\prime \prime}\left(\sigma_{t}^{\lambda^{\prime \prime}}(B)\right)=\Delta_{\lambda^{\prime \prime}}^{t \prime} \lambda^{\prime \prime}(B),\left(B \in D\left(\lambda^{\prime \prime}\right), t \in \mathbf{R}\right), \\
& \sigma_{t}^{\lambda^{\prime \prime}}\left(D\left(\lambda^{\prime}\right)\right)=D\left(\lambda^{\prime}\right) \text { and } \lambda^{\prime}\left(\sigma_{t}^{\lambda^{\prime \prime}}(K)\right)=\Delta_{\lambda^{\prime \prime}}^{i t} \lambda^{\prime}(K),\left(K \in D\left(\lambda^{\prime}\right), t \in \mathbf{R}\right) .
\end{aligned}
$$

In fact, the statement (5.9) follows from Lemma 5.1, (3) and

$$
\begin{aligned}
\sigma_{t}^{\lambda^{\prime \prime}}(\boldsymbol{B}) \lambda^{\prime}(\boldsymbol{K}) & =\Delta_{\lambda^{\prime \prime}}^{i t} B \Delta_{\lambda^{\prime \prime}}^{-i t} \lambda^{\prime}(\boldsymbol{K}) \\
& =\Delta_{\lambda^{\prime \prime}}^{i t} B \lambda^{\prime}\left(\sigma_{-t}^{\lambda^{\prime \prime}}(\boldsymbol{K})\right) \quad(\text { by } 5.8) \\
& =\Delta_{\lambda^{\prime \prime}}^{i t} \sigma_{-t}^{\lambda^{\prime \prime}}(\boldsymbol{K}) \lambda^{\prime \prime}(\boldsymbol{B}) \\
& =K \Delta_{\lambda^{\prime \prime}}^{i t} \lambda^{\prime \prime}(\boldsymbol{B})
\end{aligned}
$$

for all $B \in D\left(\lambda^{\prime \prime}\right), K \in D\left(\lambda^{\prime}\right) \cap D\left(\lambda^{\prime}\right)^{*}$ and $t \in \mathbb{R}$. The statement (5.10) follows from $\lambda^{\prime \prime \prime}=\lambda^{\prime}$ and (5.9).

We have the almost same results as Lemma 5.1, 5.2 and (5.4) (5.10) for $\lambda^{c c}$.

\section{Lemma 5. 3. Suppose}

(iii) ${ }^{\prime} \quad \lambda^{c}\left(\left(D\left(\lambda^{c}\right) \cap D\left(\lambda^{c}\right)^{*}\right)^{2}\right)$ is total in $\mathscr{H}$.

Then the following statements hold :

(1) $\lambda^{c}\left(D\left(\lambda^{c}\right) \cap D\left(\lambda^{c}\right)^{*}\right)$ is a right Hilbert subalgebra of the right Hilbert algebra $\lambda^{\prime}\left(D\left(\lambda^{\prime}\right) \cap D\left(\lambda^{\prime}\right)^{*}\right)$.

(2) $\lambda^{c c}\left(D\left(\lambda^{c c}\right) \cap D\left(\lambda^{c c}\right)^{*}\right)$ is an achieved left Hilbert algebra in $\mathscr{H}$ containing $\lambda^{\prime \prime}\left(D\left(\lambda^{\prime \prime}\right) \cap D\left(\lambda^{\prime \prime}\right) *\right)$.

(3) Let $S_{\lambda^{c c}}$ be the closure of the involution $\lambda^{c c}(A) \rightarrow \lambda^{c c}\left(A^{*}\right)\left(A \in D\left(\lambda^{c c}\right) \cap\right.$ 
$\left.D\left(\lambda^{c c}\right)^{*}\right)$ and let $S_{\lambda} c c=J_{\lambda} c c \Delta_{\lambda c c}^{1 / 2}$ be the polar decomposition of $S_{\lambda} c c$. Then, $S_{\lambda} \subset S_{\lambda^{\prime \prime}} \subset$ $S_{\lambda} c c$.

(4) $J_{\lambda} c c\left(\mathscr{M}_{\mathrm{w}}^{\prime}\right)^{\prime} J_{\lambda} c c=\mathscr{M}_{\mathrm{w}}^{\prime}$.

(5) $\sigma_{t}^{\lambda^{c c}}(\boldsymbol{A}) \equiv \Delta_{\lambda^{c c}}^{i t} A \Delta_{\lambda^{c c}}^{-i t} \in\left(\mathscr{M}_{\mathrm{w}}^{\prime}\right)^{\prime}\left(A \in\left(\mathscr{M}_{\mathrm{w}}^{\prime}\right)^{\prime}, t \in \mathbf{R}\right)$, $\sigma_{t}^{\lambda^{c c}}\left(\boldsymbol{D}\left(\lambda^{c c}\right)\right)=\boldsymbol{D}\left(\lambda^{c c}\right)$ and $\lambda^{c c}\left(\sigma_{t}^{\lambda^{c c}}(B)\right)=\Delta_{\lambda^{c c}}^{i t} \lambda^{c c}(B)$ $\left(B \in D\left(\lambda^{c c}\right), t \in \mathbf{R}\right)$, $\sigma_{t}^{\lambda^{c c}}\left(D\left(\lambda^{c c}\right) \cap D\left(\lambda^{c c}\right)^{*}\right)=D\left(\lambda^{c c}\right) \cap D\left(\lambda^{c c}\right)^{*}$ and $\lambda^{c c}\left(\sigma_{t}^{\lambda^{c c}}(B)\right)=\Delta_{\lambda^{c c}}^{i t} \lambda^{c c}(B)\left(B \in D\left(\lambda^{c c}\right) \cap D\left(\lambda^{c c}\right)^{*}, t \in \mathbf{R}\right)$.

(6) $\sigma_{t}^{\lambda^{c c}}(C) \equiv \Delta_{\lambda^{c c}}^{i t} C \Delta_{\lambda^{c c}}^{-i t} \in \mathscr{M}_{\mathrm{w}}^{\prime}\left(C \in \mathscr{M}_{\mathrm{w}}^{\prime}, t \in \mathbf{R}\right)$, $\sigma_{t}^{\lambda^{c c}}\left(D\left(\lambda^{c c c}\right)\right)=D\left(\lambda^{c c c}\right)$ and $\lambda^{c c c}\left(\sigma_{t}^{\lambda^{c c}}(K)\right)=\Delta_{\lambda^{c c c}}^{i t} \lambda^{c c c}(K)$ $\left(K \in D\left(\lambda^{c c c}\right), t \in \mathbf{R}\right)$, $\sigma_{t}^{\lambda^{c c}}\left(D\left(\lambda^{c c c}\right) \cap D\left(\lambda^{c c c}\right)^{*}\right)=D\left(\lambda^{c c c}\right) \cap D\left(\lambda^{c c c}\right) *$ and $\lambda^{c c c}\left(\sigma_{t}^{\lambda^{c c}}(K)\right)=\Delta_{\lambda^{c c}}^{i t} \lambda^{c c c}(K)\left(K \in D\left(\lambda^{c c c}\right) \cap D\left(\lambda^{c c c}\right)^{*}, t \in \mathbf{R}\right)$.

Remark. Let $\xi_{0}$ be as in Example 4.6. As seen in Example 4.6, (6), ( $\mathscr{M}, \lambda_{\xi_{0}}$, $\left.\lambda \bar{\xi}_{0}^{c}\right)$ is a cyclic and separating system, then $\lambda_{\xi_{0}}^{c c}=\lambda_{\xi_{0}}^{\prime \prime}$, and so the right Hilbert algebras $\lambda^{c}\left(D\left(\lambda^{c}\right) \cap D\left(\lambda^{c}\right)^{*}\right)$ and $\lambda^{\prime}\left(D\left(\lambda^{\prime}\right) \cap D\left(\lambda^{\prime}\right)^{*}\right)$ are equivalent. But, in general we don't know whether their right Hilbert algebras are equivalent, or not.

By Lemma 5.3 the unitary groups $\left\{\Delta_{\lambda^{\prime \prime}}^{i t}\right\}_{t \in \mathbf{R}}$ and $\left\{\Delta_{\lambda^{c c}}^{i t}\right\}_{\mathrm{t} \in \mathbf{R}}$ implement one-parameter groups $\left\{\sigma_{t}^{\lambda^{\prime \prime}}\right\}_{t \in \mathbf{R}}$ and $\left\{\sigma_{t}^{\lambda^{2 c}}\right\}_{t \in \mathbf{R}}$ of automorphisms of the von Neumann algebra $\left(\mathscr{M}_{\mathrm{w}}^{\prime}\right)^{\prime}$, respectively. But, we don't know how they act on the $O^{*}$-algebra $\mathscr{M}$, and so we need to define the following notions :

Definition 5. 4. A triple $\left(\mathscr{M}, \lambda, \lambda^{\prime}\right)$ is said to be a standard system if it satisfies the above conditions (i) (iii) and the following conditions (iv) and (v):

(iv) $\Delta_{\lambda^{\prime \prime}}^{i t} \mathscr{D} \subset \mathscr{D}$ and $\Delta_{\lambda^{\prime \prime}}^{i t} \mathscr{M} \Delta_{\lambda^{\prime \prime}}^{i t}=\mathscr{M}(t \in \mathbf{R})$.

(v) $\sigma_{t}^{\lambda^{\prime \prime}}\left(D(\lambda) \cap D(\lambda)^{\dagger}\right)=D(\lambda) \cap D(\lambda)^{\dagger}(t \in \mathbf{R})$.

A triple $\left(\mathscr{M}, \lambda, \lambda^{c}\right)$ is said to be an essentially standard system if it satisfies the conditions (i), (ii), (iii)' and the following condition (iv)'

(iv) $\Delta_{\lambda^{c c}}^{i t} \mathscr{D} \subset \mathscr{D}$ and $\Delta_{\lambda^{c c}}^{i t} \mathscr{M} \Delta_{\lambda^{c c}}^{-i t}=\mathscr{M}(t \in \mathbf{R})$.

Furthermore, if

$$
(\mathrm{v})^{\prime} \quad \sigma_{t}^{\lambda^{c c}}\left(D(\lambda) \cap D(\lambda)^{\dagger}\right)=D(\lambda) \cap D(\lambda)^{\dagger}(t \in \mathbf{R}),
$$


then $\left(\mathscr{M}, \lambda, \lambda^{c}\right)$ is said to be a standard system.

Theorem 5. 5. Suppose $\left(\mathscr{M}, \lambda, \lambda^{\prime}\right)$ is a standard system. Then the following statements hold :

(1) $S_{\lambda}=S_{\lambda^{\prime \prime}}$.

(2) $\sigma_{t}^{\lambda}(X) \equiv \Delta_{\lambda}^{i t} X \Delta_{\lambda}^{-i t}=\sigma_{t}^{\lambda^{\prime \prime}}(X)(X \in \mathscr{M}, t \in \mathbb{R})$ and $\left\{\sigma_{t}^{\lambda}\right\}_{t \in \mathbf{R}}$ is a one-parameter group of *-automorphisms of $\mathscr{M}$.

(3) $\lambda$ satisfies the $K M S$-condition with respect to $\left\{\sigma_{t}^{\lambda}\right\}_{t \in \mathbf{R}}$, that is, for each $X$, $Y \in D(\lambda) \cap D(\lambda)^{\dagger}$ there exists an element $f_{X, Y}$ of $A(0,1)$ such that

$$
f_{X, Y}(t)=\left(\lambda\left(\sigma_{t}^{\lambda}(\boldsymbol{X})\right), \lambda(Y)\right) \text { and } f_{X, Y}(t+i)=\left(\lambda\left(Y^{\dagger}\right) ; \lambda\left(\sigma_{t}^{\lambda}\left(X^{\dagger}\right)\right)\right)
$$

for all $t \in \mathbb{R}$, where $A(0,1)$ is the set of all complex-valued functions, bounded and continuous on $0 \leq \operatorname{Im} z \leq 1$ and analytic in the interior.

Proof. Take arbitrary $X, Y \in D(\lambda) \cap D(\lambda)^{\dagger}$. By (5.3) there exists sequences $\left\{X_{n}\right\}$ and $\left\{Y_{n}\right\}$ in $D\left(\lambda^{\prime \prime}\right) \cap D\left(\lambda^{\prime \prime}\right) *$ such that

$$
\begin{array}{ll}
\lim _{n \rightarrow \infty} \lambda^{\prime \prime}\left(X_{n}\right)=\lambda(X), & \lim _{n \rightarrow \infty} \lambda^{\prime \prime}\left(X_{n}^{*}\right)=\lambda\left(X^{\dagger}\right), \\
\lim _{n \rightarrow \infty} \lambda^{\prime \prime}\left(Y_{n}\right)=\lambda(Y), & \lim _{n \rightarrow \infty} \lambda^{\prime \prime}\left(Y_{n}^{*}\right)=\lambda\left(Y^{\dagger}\right) .
\end{array}
$$

By ( $[22]$ Theorem 10.17) and (5.6), for each $n \in \mathbb{N}$ there exists an element $f_{n} \in$ $A(0,1)$ such that

$$
\begin{gathered}
f_{n}(t)=\left(\lambda^{\prime \prime}\left(\sigma_{t}^{\lambda^{\prime \prime}}\left(\boldsymbol{X}_{n}\right)\right) \mid \lambda^{\prime \prime}\left(Y_{n}\right)\right)=\left(\Delta_{\lambda^{\prime \prime}}^{t t} \lambda^{\prime \prime}\left(\boldsymbol{X}_{n}\right) \mid \lambda^{\prime \prime}\left(Y_{n}\right)\right), \\
f_{n}(t+i)=\left(\lambda^{\prime \prime}\left(Y_{n}^{*}\right) \mid \lambda^{\prime \prime}\left(\sigma_{t}^{\lambda^{\prime \prime}}\left(\boldsymbol{X}_{n}^{*}\right)\right)\right)=\left(\lambda^{\prime \prime}\left(Y_{n}^{*}\right) \mid \Delta_{\lambda^{\prime \prime}}^{u \prime \prime} \lambda^{\prime \prime}\left(X_{n}^{*}\right)\right)
\end{gathered}
$$

for all $t \in \mathbb{R}$. By the condition (v) in Definition 5.4, $\lambda\left(\sigma_{t}^{\lambda^{\prime \prime}}(X)\right)$ is well-defined and

$$
\lambda\left(\sigma_{t}^{\lambda^{\prime \prime}}(X)\right)=\Delta_{\lambda^{\prime \prime}}^{i t} \lambda(X)(t \in \mathbb{R}) .
$$

In fact, this follows from the equality :

$$
\begin{aligned}
\left(\lambda\left(\sigma_{t}^{\lambda^{\prime \prime}}(\boldsymbol{X})\right) \mid \lambda^{\prime}\left(K_{1}^{*} K_{2}\right)\right) & \left.=\overline{\left(\sigma_{t}^{\lambda^{\prime \prime}}(\boldsymbol{X})\right.} \lambda^{\prime}\left(\boldsymbol{K}_{1}\right) \mid \lambda^{\prime}\left(\boldsymbol{K}_{2}\right)\right) \\
& =\left(\Delta_{\lambda^{\prime \prime}}^{i t} \bar{X} \lambda^{\prime}\left(\sigma_{-t}^{\lambda^{\prime \prime}}\left(\boldsymbol{K}_{1}\right)\right) \mid \lambda^{\prime}\left(\boldsymbol{K}_{2}\right)\right) \\
& =\left(\boldsymbol{K}_{1} \Delta_{\lambda^{\prime \prime}}^{t \prime} \lambda(\boldsymbol{X}) \mid \lambda^{\prime}\left(\boldsymbol{K}_{2}\right)\right) \\
& =\left(\Delta_{\lambda^{\prime \prime}}^{t t} \lambda(\boldsymbol{X}) \mid \lambda^{\prime}\left(\boldsymbol{K}_{1}^{*} K_{2}\right)\right)
\end{aligned}
$$


for all $K_{1}, K_{2} \in D\left(\lambda^{\prime}\right) \cap D\left(\lambda^{\prime}\right)^{*}$. By (5.11), (5.12) and (5.13) we have

$$
\begin{aligned}
& \sup _{t \in \mathbf{R}}\left|f_{n}(t)-\left(\lambda\left(\sigma_{t}^{\lambda^{\prime \prime}}(X)\right) \mid \lambda(Y)\right)\right| \\
& \leq\left\|\lambda^{\prime \prime}\left(X_{n}\right)-\lambda(X)\right\|\left\|\lambda^{\prime \prime}\left(Y_{n}\right)\right\|+\|\lambda(X)\|\left\|\lambda^{\prime \prime}\left(Y_{n}\right)-\lambda(Y)\right\| \underset{n \rightarrow \infty}{\longrightarrow} 0, \\
& \sup _{t \in \mathbb{R}}\left|f_{n}(t+i)-\left(\lambda\left(Y^{\dagger}\right) \mid \lambda\left(\sigma_{t}^{\lambda^{\prime \prime}}\left(X^{\dagger}\right)\right)\right)\right| \\
& \leq\left\|\lambda^{\prime \prime}\left(Y_{n}^{*}\right)-\lambda\left(Y^{\dagger}\right)\right\|\left\|\lambda^{\prime \prime}\left(X_{n}^{*}\right)\right\|+\left\|\lambda\left(Y^{\dagger}\right)\right\|\left\|\lambda^{\prime \prime}\left(X_{n}^{*}\right)-\lambda\left(X^{\dagger}\right)\right\| \underset{n \rightarrow \infty}{\longrightarrow} 0 .
\end{aligned}
$$

Hence, there exists an element $f_{X, Y}$ of $A(0,1)$ such that

$$
f_{X, Y}(t)=\left(\lambda\left(\sigma_{t}^{\lambda^{\prime \prime}}(X)\right) \mid \lambda(Y)\right) \text { and } f_{X, Y}(t+i)=\left(\lambda\left(Y^{\dagger}\right) \mid \lambda\left(\sigma_{t}^{\lambda^{\prime \prime}}\left(X^{\dagger}\right)\right)\right)(t \in \mathbf{R}) \text {. }
$$

We next show $S_{\lambda}=S_{\lambda^{\prime \prime}}$. Let $\mathscr{K}$ be the closure of $\left\{\lambda(X) ; X^{\dagger}=X \in D(\lambda) \cap D(\lambda)^{\dagger}\right\}$ in $\mathscr{H}$. Then $\mathscr{K}$ is a closed real subspace of $\mathscr{H}$. Since $\lambda\left(D(\lambda) \cap D(\lambda)^{\dagger}\right) \subset \mathscr{K}+i \mathscr{K}$ and it is dense in $\mathscr{H}$, we have $(\mathscr{K}+i \mathscr{K})^{\perp}=\{0\}$. Furthermore, we have $\mathscr{K} \cap i \mathscr{K}=\{0\}$. In fact, take an arbitrary $\xi \in \mathscr{K} \cap i \mathscr{K}$. Then there exist sequences $\left\{A_{n}\right\}$ and $\left\{B_{n}\right\}$ in $D(\lambda) \cap D(\lambda)^{\dagger}$ such that $A_{n}^{\dagger}=A_{n}, B_{n}^{\dagger}=B_{n}, \lim _{n \rightarrow \infty} A_{n}=\xi$ and $\lim _{n \rightarrow \infty} B_{n}=-i \xi$, and then we have

$$
\begin{aligned}
\left(\xi \mid \lambda^{\prime}\left(K_{1}^{*} K_{2}\right)\right) & =\lim _{n \rightarrow \infty}\left(\lambda\left(A_{n}\right) \mid \lambda^{\prime}\left(K_{1}^{*} K_{2}\right)\right) \\
& =\lim _{n \rightarrow \infty}\left(\overline{A_{n}} \lambda^{\prime}\left(K_{1}\right) \mid \lambda^{\prime}\left(K_{2}\right)\right) \\
& =\lim _{n \rightarrow \infty}\left(\lambda^{\prime}\left(K_{1}\right) \mid K_{2} \lambda\left(A_{n}\right)\right) \\
& =\left(\lambda^{\prime}\left(K_{2}^{*} K_{1}\right) \mid \xi\right)
\end{aligned}
$$

for all $K_{1}, K_{2} \in D\left(\lambda^{\prime}\right) \cap D\left(\lambda^{\prime}\right)^{*}$, and so

$$
\begin{aligned}
-i\left(\xi \mid \lambda^{\prime}\left(K_{1}^{*} K_{2}\right)\right) & =\lim _{n \rightarrow \infty}\left(\lambda\left(B_{n}\right) \mid \lambda^{\prime}\left(K_{1}^{*} K_{2}\right)\right) \\
& =\left(\lambda^{\prime}\left(K_{2}^{*} K_{1}\right) \mid-i \xi\right) \\
& =i\left(\lambda^{\prime}\left(K_{2}^{*} K_{1}\right) \mid \xi\right) \\
& =i\left(\xi \mid \lambda^{\prime}\left(K_{1}^{*} K_{2}\right)\right) .
\end{aligned}
$$

Hence, $\left(\xi \mid \lambda^{\prime}\left(K_{1}^{*} K_{2}\right)\right)=0$ for all $K_{1}, K_{2} \in D\left(\lambda^{\prime}\right) \cap D\left(\lambda^{\prime}\right) *$. Since $\lambda^{\prime}\left(\left(D\left(\lambda^{\prime}\right) \cap\right.\right.$ $\left.D\left(\lambda^{\prime}\right)^{*}\right)^{2}$ ) is total in $\mathscr{H}$, we have $\xi=0$. Therefore, it follows from [17] that $S_{\lambda}$ equals the closed operator $S$ defined by 


$$
S(\xi+i \eta)=\xi-i \eta(\xi, \eta \in \mathscr{K})
$$

Furthermore, by (5.14) the one-parameter group $\left\{\Delta_{\lambda^{\prime \prime}}^{i t}\right\}_{t \in \mathbf{R}}$ of unitary operators satisfies the KMS-condition with respect to $\mathscr{K}$ in the sense of ([17] Definition 3.4) and by (v) in Definition $5.4 \Delta_{\lambda^{\prime \prime}}^{i t} \mathscr{K} \subset \mathscr{K}$ for all $t \in \mathbf{R}$, and so it follows from ([17] Theorem 3.8) and (5.15) that $\Delta_{\lambda^{\prime \prime}}^{i t}=\Delta_{\lambda}^{i t}$ for all $t \in \mathbf{R}$. Therefore, $S_{\lambda^{\prime \prime}}=S_{\lambda}$, and so by (v) in Definition 5.3 and (5.14) $\lambda$ satisfies the KMS-condition with respect to the one-parameter group $\left\{\sigma_{t}^{\lambda}\right\}_{t \in \mathbf{R}}$ of $*$-automorphisms of $\mathscr{M}$. This completes the proof.

Theorem 5. 6. Suppose $\left(\mathscr{M}, \lambda, \lambda^{c}\right)$ is a standard system. Then $\left(\mathscr{M}, \lambda, \lambda^{\prime}\right)$ is a standard system satisfying $S_{\lambda}=S_{\lambda^{\prime \prime}}=S_{\lambda^{c c}}$.

Proof. We can show $S_{\lambda}=S_{\lambda}$ cc in the same way as in Theorem 5. 5, and by Lemma 5. 3, (3) $S_{\lambda}=S_{\lambda^{\prime \prime}}=S_{\lambda^{c c}}$. Therefore, $\left(\mathscr{M}, \lambda, \lambda^{\prime}\right)$ is a standard system. This completes the proof.

Remark 5. 7. Suppose $\lambda^{\sigma}\left(\left(D\left(\lambda^{\sigma}\right) \cap D\left(\lambda^{\sigma}\right)^{*}\right)^{2}\right)$ is total in $\mathscr{H}$. Then the closed operator $S_{\lambda^{\sigma \sigma}}$ is defined as the closure of the involution $\lambda^{\sigma \sigma}(A) \rightarrow \lambda^{\sigma \sigma}\left(A^{*}\right)$ and $S_{\lambda^{\sigma \sigma}} \subset S_{\lambda^{\prime \prime}}$ $\subset S_{\lambda}{ }^{c c}$, but $S_{\lambda}$ oo and $S_{\lambda}$ don't have any relation in general. So, we don't consider the standardness of $\left(\mathscr{M}, \lambda, \lambda^{\sigma}\right)$.

We next show that if $\left(\mathscr{M}, \lambda, \lambda^{c}\right)$ is an essentially standard system, then there exists a cyclic generalized vector $\lambda_{e}$ for $\mathscr{M}$ which is an extension of $\lambda$ such that $(\mathscr{M}$, $\left.\lambda_{e}, \lambda_{e}^{c}\right)$ is a standard system.

Theorem 5. 8. Suppose $\left(\mathscr{M}, \lambda, \lambda^{c}\right)$ is an essentially standard system and then put

$$
\left\{\begin{array}{l}
D\left(\lambda_{e}\right)=\left\{\sum_{k} Y_{k} X_{k} ; Y_{k} \in \mathscr{M}, X_{k} \in D(\bar{\lambda})\right\} \\
\lambda_{e}\left(\sum_{k} Y_{k} X_{k}\right)=\sum_{k} Y_{k} \bar{\lambda}\left(X_{k}\right)
\end{array}\right.
$$

where

$$
\left\{\begin{array}{l}
D(\bar{\lambda})=\left\{X \in \mathscr{M} ;{ }^{\exists}\left\{A_{\gamma}\right\} \subset D\left(\lambda^{c c}\right) \text { s.t. } A_{\gamma} \xi \rightarrow X \xi, \quad \forall \xi \in \mathscr{D} \text { and } \lambda^{c c}\left(A_{\gamma}\right) \rightarrow \xi_{X} \in \mathscr{D}\right\} \\
\bar{\lambda}(X)=\xi_{X}, X \in D(\bar{\lambda}) .
\end{array}\right.
$$

Then $\lambda_{e}$ is a cyclic generalized vector for $\mathscr{M}$ such that
(1) $\lambda_{e} \supset \lambda$,
(2) $\lambda_{e}^{c}=\lambda^{c}$,
(3) $\left(\mathscr{M}, \lambda_{e}, \lambda_{e}^{c}\right)$ is a standard system. 
Proof. Since $\lambda^{c}\left(\left(D\left(\lambda^{c}\right) \cap D\left(\lambda^{c}\right)^{*}\right)^{2}\right)$ is total in $\mathscr{H}$, it follows that $\bar{\lambda}$ is a well-defined linear map of the vector space $D(\bar{\lambda})$ into $\mathscr{D}$. Take arbitrary $K \in D\left(\lambda^{c}\right)$ and $X \in D(\bar{\lambda})$. Then there exists a net $\left\{A_{\gamma}\right\}$ in $D\left(\lambda^{c c}\right)$ such that $A_{\gamma} \xi \rightarrow X \xi$ for each $\xi \in \mathscr{D}$ and $\lambda^{c c}\left(A_{\gamma}\right) \rightarrow \bar{\lambda}(X)$. Since $\lambda^{c}(K) \in \mathscr{D}$, we have

$$
X \lambda^{c}(K)=\lim _{\gamma} A_{\gamma} \lambda^{c}(K)=\lim _{\gamma} K \lambda^{c c}\left(A_{\gamma}\right)=K \bar{\lambda}(X),
$$

which implies that $\lambda_{e}$ is a generalized vector for $\mathscr{M}$ such that

$$
\left(\sum_{k} Y_{k} X_{k}\right) \lambda^{c}(K)=K \lambda_{e}\left(\sum_{k} Y_{k} X_{k}\right), \sum_{k} Y_{k} X_{k} \in D\left(\lambda_{e}\right) .
$$

Hence, $K \in D\left(\lambda_{e}^{c}\right)$ and $\lambda_{e}^{c}(K)=\lambda^{c}(K)$. By (5.3) we have $\bar{\lambda} \supset \lambda$, and so $\lambda_{e} \supset \lambda$. Hence, $\lambda_{e}^{c} \subset \lambda^{c}$. Thus we have $\lambda_{e}^{c}=\lambda^{c}$ and so $\left(\mathscr{M}, \lambda_{e}, \lambda_{e}^{c}\right)$ is an essentially standard system. We finally show $\sigma_{t}^{\lambda^{c c}}\left(D\left(\lambda_{e}\right) \cap D\left(\lambda_{e}\right)^{\dagger}\right) \subset D\left(\lambda_{e}\right) \cap D\left(\lambda_{e}\right)^{\dagger}$ for each $t \in \mathbf{R}$. Take an arbitrary $X \in D\left(\lambda_{e}\right) \cap D\left(\lambda_{e}\right)^{\dagger}$. Then, since $\lambda_{e}^{\prime \prime} \subset \lambda_{e}^{c c}=\lambda^{c c}$, it follows from (5.3) that there exists a sequence $\left\{X_{n}\right\}$ in $D\left(\lambda^{c c}\right) \cap D\left(\lambda^{c c}\right)^{*}$ such that $X_{n} \underset{t_{s}^{*}}{\rightarrow} X$ (that is, $\lim _{n \rightarrow \infty} X_{n} \xi=X \xi$ and $\left.\lim _{n \rightarrow \infty} X_{n}^{*} \xi=X^{\dagger} \xi, \quad \forall \xi \in \mathscr{D}\right), \lim _{n \rightarrow \infty} \lambda^{c c}\left(X_{n}\right)=\lambda_{e}(X)$ and $\lim _{n \rightarrow \infty} \lambda^{c c}\left(X_{n}^{*}\right)=\lambda_{e}\left(X^{\dagger}\right)$. Hence it follows from Lemma 5. 3, (5) that for each $t$ $\stackrel{n \rightarrow \infty}{\in}\left\{\sigma_{t}^{\lambda^{c c}}\left(X_{n}\right)\right\} \subset D\left(\lambda^{c c}\right) \cap D\left(\lambda^{c c}\right)^{*}, \Delta_{\lambda}^{i t c c} \mathscr{D} \subset \mathscr{D}, \sigma_{t}^{\lambda^{c c}}\left(X_{n}\right) \underset{t_{s}^{*}}{\rightarrow \sigma_{t}^{\lambda^{c c}}}(X), \lim _{n \rightarrow \infty} \lambda^{c c}\left(\sigma_{t}^{\lambda^{c c}}\left(X_{n}\right)\right)$ $=\lim _{n \rightarrow \infty} \Delta_{\lambda^{i t}}^{i t} \lambda^{c c}\left(X_{n}\right)=\Delta_{\lambda}^{t t} \lambda_{e}(X)$ and $\lim _{n \rightarrow \infty} \lambda^{c c}\left(\left(X_{n}\right)^{*}\right)=\Delta_{\lambda}^{u t c c} \lambda_{e}\left(X^{\dagger}\right)$, which implies that $\sigma_{t}^{\lambda_{t c}^{n c \infty}}(X) \in D\left(\lambda_{e}\right) \cap D\left(\lambda_{e}\right)^{\dagger}$ and $\lambda_{e}^{n \rightarrow \infty}\left(\sigma_{t}^{\lambda^{c c}}(X)\right)=\Delta_{\lambda}^{t c c} \lambda_{e}(X)$. Therefore, we have $\sigma_{t}^{\lambda^{c c}}\left(D\left(\lambda_{e}\right) \cap D\left(\lambda_{e}\right)^{\dagger}\right) \subset D\left(\lambda_{e}\right) \cap D\left(\lambda_{e}\right)^{\dagger}$ for each $t \in \mathbf{R}$, which implies $\left(\mathscr{M}, \lambda_{e}, \lambda_{e}^{c}\right)$ is a standard system. This completes the proof.

Remark 5.9. Suppose ( $\left.\mathscr{M}, \lambda, \lambda^{\prime}\right)$ satisfies the conditions (i) $\sim($ iv) and it doesn't satisfy the condition (v). Then we don't know whether the same result as Theorem 5. 8 holds.

Weakening the condition (iv)' in Definition 5.4, we define the notion of modular systems which is able to apply the unbounded Tomita-Takesaki theory to more examples.

Definition 5. 10. $A$ system $\Gamma=\left(\mathscr{M}, \lambda, \lambda^{c}\right)$ is said to be modular if the conditions (i), (ii) and (iii)' in Definition 5.4 and the following condition (iv)" hold:

(iv)" There exists a dense subspace $\mathscr{E}$ of $\left(\mathscr{D}, t_{\mathscr{M}}\right)$ such that

(iv) ${ }_{1}^{\prime \prime} \quad \lambda\left(D(\lambda) \cap D(\lambda)^{\dagger}\right) \subset \mathscr{E}$,

(iv) ${ }_{2}^{\prime \prime}\left\{\lambda^{c}\left(K_{1} K_{2}\right) ; K_{l} \in D\left(\lambda^{c}\right) \cap D\left(\lambda^{c}\right)^{*}\right.$ s.t. $\left.\lambda^{c}\left(K_{l}\right), \lambda^{c}\left(K_{l}^{*}\right) \in \mathscr{E}, i=1,2\right\}$ is total in the Hilbert space $\mathscr{D}\left(S_{\lambda^{c}}^{*}\right)$,

(iv) ${ }_{3}^{\prime \prime} \quad \mathscr{M} \mathscr{E} \subset \mathscr{E}$,

(iv) ${ }_{4}^{\prime \prime} \quad \Delta_{\lambda}^{t t} c c \mathscr{E} \subset \mathscr{E}$ for each $t \in \mathbf{R}$.

Let $\Gamma=\left(\mathscr{M}, \lambda, \lambda^{c}\right)$ be a modular system. Then there exists the maximal 
subspace $\mathscr{D}_{\Gamma}$ of $\mathscr{D}$ satisfying the above conditions (iv) ${ }_{1}^{\prime \prime} \sim(\text { iv) })_{4}^{\prime \prime}$. Since $\mathscr{D}_{\Gamma}$ is maximal, it follows that $\mathscr{M}_{\mathrm{w}}^{\prime} \mathscr{D}_{\Gamma} \subset \mathscr{D}_{\Gamma}$, which implies by ([7] Theorem 3. 3) that

$$
\mathscr{U}(\Gamma) \equiv\left\{X \in \mathscr{L}^{\dagger}\left(\mathscr{D}_{\Gamma}\right) ; \bar{X} \text { is affiliated with }\left(\mathscr{M}_{\mathrm{w}}^{\prime}\right)^{\prime}\right\}
$$

is a generalized von Neumann algebra on $\mathscr{D}_{\Gamma}$ over $\left(\mathscr{M}_{\mathrm{w}}^{\prime}\right)^{\prime}$ generated by $\mathscr{M}\left\lceil\mathscr{D}_{\Gamma}\right.$ and the $O^{*}$-algebra $\mathscr{L}(\Gamma)$ on $\mathscr{D}_{\Gamma}$ generated by $\left\{\Delta_{\lambda^{c c}}^{i t} \mathscr{M}_{\lambda^{c c}}^{-i t}\left\lceil\mathscr{D}_{\Gamma} ; t \in \mathbb{R}\right\}\right.$ is a closed $O^{*}$ subalgebra of $\mathscr{U}(\Gamma)$. Furthermore, $\left\{\sigma_{t}^{\lambda^{c c}}\right\}_{t \in \mathbf{R}}$ is a one-parameter group of $*$ automorphisms of $\mathscr{L}(\Gamma)$ and $\mathscr{U}(\Gamma)$. For modular systems we have the following

Theorem 5。且目。 Suppose $\Gamma=\left(\mathscr{M}, \lambda, \lambda^{c}\right)$ is a modular system and then put

$$
\begin{aligned}
& D\left(\lambda_{s}\right)=\left\{\sum_{k} Y_{k} X_{k} ; Y_{k} \in \mathscr{M}, X_{k} \in D(\bar{\lambda})\right\}, \\
& \lambda_{s}\left(\sum_{k} Y_{k} X_{k}\right)=\sum_{k} Y_{k} \bar{\lambda}\left(X_{k}\right),
\end{aligned}
$$

where

$$
\begin{aligned}
D(\bar{\lambda})= & \left\{X \in \mathscr{U}(\Gamma) ;{ }^{\exists}\left\{A_{\gamma}\right\} \subset D\left(\lambda^{c c}\right) \text { s.t. } A_{\gamma} \xi \rightarrow X \xi, \quad \forall \xi \in \mathscr{D}_{\Gamma}\right. \\
& \text { and } \left.\lambda^{c c}\left(A_{\gamma}\right) \rightarrow \xi_{X} \in \mathscr{D}_{\Gamma}\right\}, \\
\bar{\lambda}(X)= & \xi_{X}, X \in \mathscr{D}(\bar{\lambda}) .
\end{aligned}
$$

Then $\lambda_{s}$ is a cyclic generalized vector for $\mathscr{U}(\Gamma)$ such that

(1) $D\left(\lambda_{s}^{c}\right)=\left\{K \in D\left(\lambda^{c}\right) ; \lambda^{c}(K) \in \mathscr{D}_{\Gamma}\right\}$ and $D\left(\lambda_{s}^{c c}\right) \cap D\left(\lambda_{s}^{c c}\right) *=D\left(\lambda^{c c}\right) \cap D\left(\lambda^{c c}\right)^{*}$;

(2) $\quad\left(\mathscr{U}(\Gamma), \lambda_{s}, \lambda_{s}^{c}\right)$ is a standard system;

(3) $\quad\left(\mathscr{L}(\Gamma), \lambda_{s} \Gamma \mathscr{L}(\Gamma),\left(\lambda_{s} \Gamma \mathscr{L}(\Gamma)\right)^{c}\right)$ is a standard system, where $D\left(\lambda_{s}\right.$ $\lceil\mathscr{L}(\Gamma))=D\left(\lambda_{s}\right) \cap \mathscr{L}(\Gamma)$ and $\left(\lambda_{s}\lceil\mathscr{L}(\Gamma))(X)=\lambda_{s}(X)\right.$ for $X \in D\left(\lambda_{s}\lceil\mathscr{L}(\Gamma))\right.$.

Proof. We can show in similar to the proof in Theorem 5.8 that $\lambda_{s}$ is a generalized vector for $\mathscr{U}(\Gamma)$. Since $\mathscr{U}(\Gamma)$ is a generalized von Neumann algebra on $\mathscr{D}_{\Gamma}$ over $\left(\mathscr{M}_{\mathrm{w}}^{\prime}\right)^{\prime}$, it follows that $\mathscr{U}(\Gamma)_{\mathrm{w}}^{\prime} \mathscr{D}_{\Gamma}=\mathscr{M}_{\mathrm{w}}^{\prime} \mathscr{D}_{\Gamma} \subset \mathscr{D}_{\Gamma}$. Since $\lambda\left(\boldsymbol{D}(\lambda) \cap \boldsymbol{D}(\lambda)^{\dagger}\right)$ $\subset \mathscr{E} \subset \mathscr{D}_{\Gamma}$, it follows from (4.4), (4.5), (5.3) and $\lambda^{\prime \prime} \subset \lambda^{c c}$ that

$$
X\left\lceil\mathscr { D } _ { \Gamma } \in \mathbb { D } ( \lambda _ { s } ) \cap \mathbb { D } ( \lambda _ { s } ) ^ { \dagger } \text { and } \lambda _ { s } \left( X\left\lceil\mathscr{D}_{\Gamma}\right)=\lambda(X)\right.\right.
$$

for all $X \in D(\lambda) \cap D(\lambda)^{\dagger}$, which implies that $\lambda_{s}\left(\left(D\left(\lambda_{s}\right) \cap D\left(\lambda_{s}\right)^{\dagger}\right)^{2}\right)$ is total in $\mathscr{H}$. We show

$$
\begin{aligned}
D\left(\lambda^{c}\right)= & \left\{K \in \mathscr{M}_{\mathrm{w}}^{\prime} ;{ }^{\exists} \xi_{K} \in \mathscr{D} \text { s.t. } K \lambda(X)=X \xi_{K}\right. \\
& \text { for all } \left.X \in D(\lambda) \cap D(\lambda)^{\dagger}\right\} .
\end{aligned}
$$


Take an arbitrary $K \in \mathscr{M}_{\mathrm{w}}^{\prime}$ such that

$$
K \lambda(X)=X \xi_{K},{ }^{\forall} X \in D(\lambda) \cap D(\lambda)^{\dagger}
$$

for some $\xi_{K} \in \mathscr{D}$. Since $Y^{\dagger} X \in D(\lambda) \cap D(\lambda)^{\dagger}$ for each $X, Y \in D(\lambda)$, it follows that

$$
Y^{\dagger} K \lambda(X)=K \lambda\left(Y^{\dagger} X\right)=Y^{\dagger} X \xi_{K}
$$

Further, since $D\left(\lambda^{c}\right) \cap D\left(\lambda^{c}\right) *$ is a nondegenerate *-subalgebra of $\mathscr{M}_{\mathrm{w}}^{\prime}$ and $\lambda(D(\lambda))$ is dense in $\mathscr{H}$, it follows that $D(\lambda) \lambda^{c}\left(D\left(\lambda^{c}\right) \cap D\left(\lambda^{c}\right)^{*}\right)=\left(D\left(\lambda^{c}\right) \cap\right.$ $\left.D\left(\lambda^{c}\right)^{*}\right) \lambda(D(\lambda))$ is total in $\mathscr{H}$, which implies by (5.18) that $K \lambda(X)=X \xi_{K}$ for all $X \in D(\lambda)$. Hence, $K \in D\left(\lambda^{c}\right)$. The converse inclusion is trivial. Thus the statement (5.17) holds. We show

$$
D\left(\lambda_{s}^{c}\right)=\left\{K \in D\left(\lambda^{c}\right) ; \lambda^{c}(K) \in \mathscr{D}_{\Gamma}\right\}
$$

Take an arbitrary $K \in D\left(\lambda^{c}\right)$ such that $\lambda^{c}(K) \in \mathscr{D}_{\Gamma}$. For each $X \in D(\bar{\lambda})$ there exists a net $\left\{A_{\gamma}\right\}$ in $D\left(\lambda^{c c}\right)$ such that $A_{\gamma} \xi \rightarrow X \xi,{ }^{\forall} \xi \in \mathscr{D}_{\Gamma}$ and $\lambda^{c c}\left(A_{\gamma}\right) \rightarrow \bar{\lambda}(X)$. Then we have

$$
X \lambda^{c}(K)=\lim _{\gamma} A_{\gamma} \lambda^{c}(K)=\lim _{\gamma} K \lambda^{c c}\left(A_{\gamma}\right)=K \bar{\lambda}(X),
$$

which implies

$$
\left(\sum_{k} Y_{k} X_{k}\right) \lambda^{c}(K)=K \lambda_{s}\left(\sum_{k} Y_{k} X_{k}\right), \sum_{k} Y_{k} X_{k} \in D\left(\lambda_{s}\right) .
$$

Hence, $K \in D\left(\lambda_{s}^{c}\right)$. Conversely, take arbitrary $K \in D\left(\lambda_{s}^{c}\right)$ and $X \in D(\lambda) \cap D(\lambda)^{\dagger}$. By (5.16) we have

$$
K \lambda(X)=K \lambda_{s}\left(X\left\lceil\mathscr{D}_{\Gamma}\right)=X \lambda_{s}^{c}(K)\right.
$$

Hence, it follows from (5.17) that $K \in D\left(\lambda^{c}\right)$ and $\lambda^{c}(K)=\lambda_{s}^{c}(K) \in \mathscr{D}_{\Gamma}$. Thus the statement (5.19) holds. It follows from (5.19) and (iv) ${ }_{2}^{\prime \prime}$ in Definition 5.10 that $D\left(\lambda_{s}^{c c}\right) \cap D\left(\lambda_{s}^{c c}\right)^{*}$ is an achieved left Hilbert algebra in $\mathscr{H}$ equivalent to $D\left(\lambda^{c c}\right) \cap$ $D\left(\lambda^{c c}\right)^{*}$. Hence, $D\left(\lambda_{s}^{c c}\right) \cap D\left(\lambda_{s}^{c c}\right)^{*}=D\left(\lambda^{c c}\right) \cap D\left(\lambda^{c c}\right)^{*}$, and so $\Delta_{\lambda_{s}^{c c}}=\Delta_{\lambda^{c c}}$. Furthermore, it follows from Lemma 5.3, (5) that $\sigma_{t}^{\lambda^{c c}}\left(D\left(\lambda_{s}\right) \cap D\left(\lambda_{s}\right)^{\dagger}\right) \subset D\left(\lambda_{s}\right) \cap D\left(\lambda_{s}\right)^{\dagger}$ for all $t \in \mathbf{R}$. Therefore, $\left(\mathscr{U}(\Gamma), \lambda_{s}, \lambda_{s}^{c}\right)$ is a standard system. Similarly, we can prove that $\left(\mathscr{L}(\Gamma), \lambda_{s}\left\lceil\mathscr{L}(\Gamma),\left(\lambda_{s}\lceil\mathscr{L}(\Gamma))^{c}\right)\right.\right.$ is a standard system. This completes the proof.

Example 5. 12. Let $\mathscr{M}$ be a closed $O^{*}$-algebra on $\mathscr{D}$ in $\mathscr{H}$ satisfying $\mathscr{M}_{\mathrm{w}}^{\prime} \mathscr{D} \subset$ $\mathscr{D}$ and $\xi_{0}$ a cyclic and separating vector for $\left(\mathscr{M}_{\mathrm{w}}^{\prime}\right)^{\prime}$. We consider when $\Gamma=\left(\mathscr{M}, \lambda \xi_{0}\right.$, $\lambda \xi_{\xi_{0}}$ ) is a standard (or modular) system, where $\lambda_{\xi_{0}}$ is a generalized vector for $\mathscr{M}$ 
defined in Example 3.2, 4.5.

(1) If the following conditions (i) (iii) hold, then $\left(\mathscr{M}, \lambda \xi_{\xi_{0}}, \lambda_{\xi_{0}}^{c}\right)$ is a standard system satisfying $\Delta_{\lambda_{\xi_{0}}}=\Delta_{\xi_{0}}$, where $\Delta_{\xi_{0}}$ is a modular operator of the achieved left Hilbert algebra $\left(\mathscr{M}_{\mathrm{w}}^{\prime}\right)^{\prime} \xi_{0}$.

(i) $\quad\left\{X^{\dagger} Y^{\dagger *} \xi_{0} ; X, Y \in D\left(\lambda_{\xi_{0}}\right) \cap D\left(\lambda_{\xi_{0}}\right)^{\dagger}\right\}$ is total in $\mathscr{H}$.

(ii) $\left\{\mathbb{K}_{1} \mathbb{K}_{2} \xi_{0} ; \mathbb{K}_{i} \in \mathscr{M}_{\mathrm{w}}^{\prime}\right.$ s.t. $\left.\mathbb{K}_{i} \xi_{0}, \mathbb{K}_{i}^{*} \xi_{0} \in \mathscr{D}, i=1,2\right\}$ is total in $\mathscr{H}$.

(iii) $\Delta_{\xi_{0}}^{i t} \mathscr{D} \subset \mathscr{D}$ and $\Delta_{\xi_{0}}^{i t} \mathscr{M} \Delta_{\xi_{0}}^{-i t}=\mathscr{M}$ for each $t \in \mathbb{R}$.

In fact, by Example $4.5\left(\mathscr{M}, \lambda_{\xi_{0}}, \lambda_{\xi_{0}}^{c}\right)$ is an essentially standard system satisfying $D\left(\lambda_{\xi_{0}}^{c c}\right)=\left(\mathscr{M}_{\mathrm{w}}^{\prime}\right)^{\prime}$ and $\lambda_{\xi_{0}}^{c c}(A)=A \xi_{0}$ for each $A \in\left(\mathscr{M}_{\mathrm{w}}^{\prime}\right)^{\prime}$ and $\Delta_{\lambda_{\xi_{0}}^{c c}}=\Delta_{\xi_{0}}$. Furthermore, since $\Delta_{\xi_{0}}^{i t} \xi_{0}=\xi_{0}$ for each $t \in \mathbb{R}$, it follows that $\xi_{0} \in \mathscr{D}\left(\sigma_{t}^{\xi_{0}}\left(X^{\dagger}\right)^{*}\right) \cap \mathscr{D}\left(\sigma_{t}^{\xi_{0}}(X)^{*}\right)$ and $\sigma_{t}^{\xi_{0}}\left(X^{\dagger}\right) * \xi_{0}, \sigma_{t}^{\xi_{0}}(X)^{*} \xi_{0} \in \mathscr{D}$ for each $X \in D\left(\lambda_{\xi_{0}}\right) \cap D\left(\lambda_{\xi_{0}}\right)^{\dagger}$ and $t \in \mathbb{R}$, which implies that $\sigma_{t}^{\xi_{0}}\left(D\left(\lambda_{\xi_{0}}\right) \cap D\left(\lambda_{\xi_{0}}\right)^{\dagger}\right) \subset D\left(\lambda_{\xi_{0}}\right) \cap D\left(\lambda_{\xi_{0}}\right)^{\dagger}$ for each $t \in \mathbb{R}$. Therefore, $\left(\mathscr{M}, \lambda_{\xi_{0}}\right.$, $\lambda\left(\xi_{0}^{c}\right)$ is a standard system.

(2) If the above conditions (i), (ii) and the following condition (iii)' hold, then $\Gamma=\left(\mathscr{M}, \lambda_{\xi_{0}}, \lambda_{\xi_{0}}^{c}\right)$ is a modular system :

(iii) $^{\prime} \quad$ There exists a dense subspace $\mathscr{E}$ of $\left(\mathscr{D}, t_{\mathscr{M}}\right)$ such that

(iii) $)_{1}^{\prime} \quad\left\{X^{\dagger *} \xi_{0} ; X \in \mathbb{D}\left(\lambda_{\xi_{0}}\right) \cap D\left(\lambda_{\xi_{0}}\right)^{\dagger}\right\} \subset \mathscr{E}$,

(iii) $)_{2}^{\prime}\left\{K_{1} K_{2} \xi_{0} ; K_{i} \in \mathscr{M}_{\mathrm{w}}^{\prime}\right.$ s.t. $\left.K_{i} \xi_{0}, K_{i}^{*} \xi_{0} \in \mathscr{E}, i=1,2\right\}$

is total in $\mathscr{H}$,

(iii) ${ }_{3}^{\prime} \quad \mathscr{M} \mathscr{E} \subset \mathscr{E}$,

(iii) ${ }_{4}^{\prime} \quad \Delta_{\xi_{0}}^{i t} \mathscr{E} \subset \mathscr{E}$ for each $t \in \mathbb{R}$.

In fact, by (iii) $)_{2}^{\prime} \mathscr{R} \equiv\left\{\mathbb{K} \xi_{0} ; K \in \mathscr{M}_{\text {w }}^{\prime}\right.$ s.t. $\left.\mathbb{K} \xi_{0}, K^{*} \xi_{0} \in \mathscr{D}_{\Gamma}\right\}$ is a right Hilbert algebra in $\mathscr{H}$ whose commutant $\mathscr{R}^{\prime}$ equals the achieved left Hilbert algebra $\left(\mathscr{M}_{\mathrm{w}}^{\prime}\right)^{\prime} \xi_{0}$ and so $\mathscr{R}^{2}$ is total in the Hilbert space $\mathscr{D}\left(S_{\xi_{0}}^{*}\right)$. Therefore, $\left(\mathscr{M}, \lambda \xi_{\xi_{0}}, \lambda \xi_{\xi_{0}}^{c}\right)$ is a modular system.

Below we give examples of standard systems and modular systems for the unbounded CCR-algebras.

Example 5. 13. Let $\mathscr{S}(\mathbb{R})$ be the Schwartz space of infinitely differentiable rapidly decreasing functions and $\left\{f_{n}\right\}_{n=0,1}, \ldots \subset \mathscr{S}(\mathbb{R})$ an orthonormal basis in the Hilbert space $L^{2}(\mathbb{R})$ of normalized Hermite functions. We denote by $L^{2}(\mathbb{R}) \otimes$ $\overline{L^{2}(\mathbb{R})}\left(\right.$ simply, $\left.\mathbb{L}^{2} \otimes \overline{L^{2}}\right)$ the Hilbert space of Hilbert-Schmidt operators on $\mathbb{L}^{2}(\mathbb{R})$ and put

$$
\pi(\mathbb{A}) T=A T, \pi^{\prime}(\mathbb{A}) T=T A
$$

for $A \in \mathscr{B}\left(L^{2}(\mathbb{R})\right)$ and $T \in L^{2} \otimes \overline{L^{2}}$. Then $\pi\left(\mathscr{B}\left(L^{2}(\mathbb{R})\right)\right)$ is a von Neumann algebra 
on $L^{2} \otimes \overline{L^{2}}$ satisfying $\pi\left(\mathscr{B}\left(L^{2}(\mathbf{R})\right)\right)^{\prime}=\pi^{\prime}\left(\mathscr{B}\left(L^{2}(\mathbf{R})\right)\right)$. Let

$$
\begin{gathered}
l_{+}^{2}=\left\{\left\{\alpha_{n}\right\} \in l^{2} ; \alpha_{n}>0, n=0,1, \cdots\right\}, \\
\Omega_{\left\{\alpha_{n}\right\}}=\sum_{n=0}^{\infty} \alpha_{n} f_{n} \otimes \overline{f_{n}}, \quad\left\{\alpha_{n}\right\} \in l_{+}^{2} .
\end{gathered}
$$

By ([7] Lemma 5. 2) $\Omega_{\left\{\alpha_{n}\right\}}$ is a cyclic and separating vector for $\pi\left(\mathscr{B}\left(L^{2}(\mathbf{R})\right)\right)$ and the modular conjugation operator $J_{\left.\Omega_{\left\{\alpha_{n}\right.}\right\}}$ equals the involution $T \in L^{2} \otimes \overline{L^{2}} \rightarrow T^{*} \in L^{2}$ $\otimes \overline{L^{2}}$ and the modular operator $\Delta_{\Omega_{\left\{a_{n}\right\}}}$ equals $\pi^{\prime}\left(\Omega_{\left\{\alpha_{n}\right\}}^{-2}\right) \pi\left(\Omega_{\left\{\alpha_{n}\right\}}^{2}\right)$, where $\mathscr{D}\left(\pi^{\prime}\left(\Omega_{\left\{\alpha_{n}\right\}}^{-2}\right\}\right)$ $=\left\{T \in L^{2} \otimes \overline{L^{2}} ; \overline{T \Omega_{\left\{\alpha_{n}\right\}}^{-2}} \in L^{2} \otimes \overline{L^{2}}\right\}$ and $\pi^{\prime}\left(\Omega_{\left\{\alpha_{n}\right\}}^{-2}\right) T=T \Omega_{\left\{\alpha_{n}\right\}}^{-2}$ for $T \in \mathscr{D}\left(\pi^{\prime}\left(\Omega_{\left\{\alpha_{n}\right\}}^{-2}\right)\right)$. Let $\mathscr{A}$ be the unbounded CCR-algebra for one degree of freedom and $\pi_{0}$ the Schrödinger representation of $\mathscr{A}$. Then $\pi_{0}(\mathscr{A})$ is a self-adjoint $O^{*}$-algebra on $\mathscr{S}(\mathbf{R})$ satisfying $\pi_{0}(\mathscr{A})_{\mathrm{w}}^{\prime}=\mathbf{C} 1$. Let $\mathscr{M}$ be an $O^{*}$-algebra on $\mathscr{S}(\mathbf{R})$ containing $\pi_{0}(\mathscr{A})$. Then we put

$$
\begin{aligned}
& \mathscr{S} \otimes \overline{L^{2}}=\left\{T \in L^{2} \otimes \overline{L^{2}} ; T L^{2}(\mathbf{R}) \subset \mathscr{S}(\mathbf{R})\right\}, \\
& \pi(X) T=X T, X \in \mathscr{M}, T \in \mathscr{S} \otimes \overline{L^{2}}
\end{aligned}
$$

and then $\pi(\mathscr{M})$ is a self-adjoint $O^{*}$-algebra on $\mathscr{S} \otimes \overline{L^{2}}$ satisfying $\pi(\mathscr{M})_{\mathrm{w}}^{\prime}=$ $\pi^{\prime}\left(\mathscr{B}\left(L^{2}(\mathbf{R})\right)\right)$ and $\left(\pi(\mathscr{M})_{\mathrm{w}}^{\prime}\right)^{\prime}=\left(\pi\left(\mathscr{B}\left(L^{2}(\mathbf{R})\right)\right)\right.$. Let $\left\{\alpha_{n}\right\} \in l_{+}^{2}$. Then $\Omega_{\left\{\alpha_{n}\right\}}$ is a cyclic vector for the von Neumann algebra $\pi\left(\mathscr{B}\left(L^{2}(\mathbf{R})\right)\right)$ but it is not a cyclic vector for the $O^{*}$-algebra $\pi(\mathscr{M})$ in general, and so we need to consider the generalized vector $\lambda_{\Omega_{\left\{a_{n}\right\}}}$ for $\pi(\mathscr{M})$ :

$$
\begin{aligned}
& D\left(\lambda_{\left.\Omega_{\left\{\alpha_{n}\right.}\right\}}\right)=\left\{\pi(X) \in \pi(\mathscr{M}) ; \Omega_{\left\{\alpha_{n}\right\}} \in \mathscr{D}\left(\pi\left(X^{\dagger}\right)^{*}\right) \text { and } \pi\left(X^{\dagger}\right)^{*} \Omega_{\left\{\alpha_{n}\right\}} \in \mathscr{S} \otimes \overline{L^{2}}\right\}, \\
& \lambda_{\Omega_{\left\{a_{n}\right\}}}(\pi(X))=\pi\left(X^{\dagger}\right) * \Omega_{\left\{\alpha_{n}\right\}}, \pi(X) \in D\left(\lambda_{\left.\Omega_{\left\{\alpha_{n}\right.}\right\}}\right) .
\end{aligned}
$$

We have the following results for the standardness (or modularity) of ( $\pi(\mathscr{M}), \lambda_{\left.\Omega_{\left\{a_{n}\right.}\right\}}$, $\lambda_{\left.\Omega_{\left\{a_{n}\right\}}\right)}^{c}$ :

(1) Suppose $\left\{\pi(Y) \pi\left(X^{\dagger}\right)^{*} \Omega_{\left\{a_{n}\right\}} ; X, Y \in D\left(\lambda_{\Omega_{\left\{a_{n}\right.}}\right) \cap D\left(\lambda_{\Omega_{\left\{a_{n}\right\}}}\right)^{\dagger}\right\}$ is total in $L^{2} \otimes$ $\overline{L^{2}}$. Then $\Gamma=\left(\pi(\mathscr{M}), \lambda_{\Omega_{\left\{\alpha_{n}\right\}}}, \lambda_{\Omega_{\left\{\alpha_{n}\right\}}}^{c}\right)$ is a modular system satisfying $\mathscr{D}_{\Gamma}=\mathscr{S} \otimes \overline{L^{2}}$.

(2) Suppose $\mathscr{M} \supset\left\{f_{n} \otimes \overline{f_{m}} ; n, m \in \mathbf{N} \cup\{0\}\right\}$. Then $\left(\pi(\mathscr{M}), \lambda_{\Omega_{\left\{a_{n}\right\}}}, \lambda_{\Omega_{\left\{\alpha_{n}\right\}}}^{c}\right)$ is a modular system.

(3) $\left(\pi\left(\mathscr{L}^{\dagger}(\mathscr{S}(\mathbf{R}))\right), \lambda_{\Omega_{\left\{\alpha_{n}\right\}}}, \lambda_{\Omega_{\left\{\alpha_{n}\right\}}}^{c}\right)$ is a standard system. In fact, since $N \equiv$ $\sum_{n=0}^{\infty}(n+1) f_{n} \otimes \overline{f_{n}} \in \pi_{0}(\mathscr{A})$ and $\mathscr{S}(\mathbf{R})=\bigcap_{k \in \mathbb{N}} \mathscr{D}\left(N^{k}\right)$, it follows that $\Omega_{\left\{\alpha_{n}\right\}}^{\imath t} \mathscr{S}(\mathbf{R}) \subset \mathscr{S}(\mathbf{R})$ for all $t \in \mathbf{R}$, which implies $\Delta_{\Omega_{\left\{a_{n}\right\}} t}^{i t} \otimes \bar{L}^{2} \subset \mathscr{S} \otimes \overline{L^{2}}$ for all $t \in \mathbf{R}$. Furthermore, it follows that 


$$
\left\{\pi^{\prime}\left(f_{n} \otimes \overline{f_{m}}\right) A\left(f_{k} \otimes \overline{f_{l}}\right) \Omega_{\left\{\alpha_{n}\right\}} ; n, m, k, l \in \mathbf{N} \cup\{0\} \text { and } A \in \mathscr{B}\left(L^{2}(\mathbf{R})\right)\right\} \subset \mathscr{S} \otimes \overline{L^{2}}
$$

and it is total in $L^{2} \otimes \overline{L^{2}}$. Hence, the statement (1) follows from Example 5. 12. If $\mathscr{M} \supset\left\{f_{n} \otimes \overline{f_{m}} ; n, m \in \mathbf{N} \cup\{0\}\right\}$, then $\left\{\pi\left(f_{n} \otimes \overline{f_{m}}\right) ; n, m \in \mathbf{N} \cup\{0\}\right\} \subset D\left(\lambda_{\Omega_{\left\{\alpha_{n}\right.}}\right) \cap$ $D\left(\lambda_{\Omega_{\left\{\alpha_{n}\right\}}}\right)^{\dagger}$ and $\left\{\pi(Y) \pi\left(X^{\dagger}\right)^{*} \Omega_{\left\{\alpha_{n}\right\}} ; X, Y \in D\left(\lambda_{\Omega_{\left\{a_{n}\right\}}}\right) \cap D\left(\lambda_{\Omega_{\left\{a_{n}\right\}}}\right)^{\dagger}\right)$ is total in $L^{2} \otimes \overline{L^{2}}$, and so the statement (2) follows from (1). Since $\Omega_{\left\{a_{n}\right\}}^{i t} \in \mathscr{L}^{\dagger}(\mathscr{S}(\mathbf{R}))$ for all $t \in \mathbf{R}$, it follows that $\Delta_{\Omega_{\left\{a_{n}\right.}}^{i t} \pi\left(\mathscr{L}^{\dagger}(\mathscr{S}(\mathbf{R})) \Delta_{\Omega_{\left\{a_{n}\right\}}}^{-i t} \subset \pi\left(\mathscr{L}^{\dagger}(\mathscr{S}(\mathbf{R}))\right.\right.$ for all $t \in \mathbf{R}$. Therefore, $\left(\pi\left(\mathscr{L}^{\dagger}(\mathscr{S}(\mathbf{R})), \lambda_{\Omega_{\left\{\alpha_{n}\right\}}}, \lambda_{\Omega_{\left\{\alpha_{n}\right\}}}\right)\right.$ is a standard system.

\section{Acknowledgements}

A. I. achnowledges gratefully the hospitality of the Institute of Theoretical Physics, University of Wroclaw, where this work was performed. The research by W. K. has been done under grant Komitet Badań Naukowych 200609101.

\section{References}

[1] Borchers, H. J., Algebraic aspects of Wightman field theory, In Statistical Mechanics and Field Theory, Lecture, 1971 ; Haifa Summer School, New York-Jerusalem-London, (1972), 31-79.

[2] Bratteli, O. and Robinson, D. W., Operator Algebras and Quantum Statistical Mechanics I, II, Springer Verlag, New York, Heidelberg, Berlin, 1979, 1981.

[ 3 ] Gudder, S. P. and Scruggs, W., Unbounded representations of *-algebras, Pacific J. Math., 70 (1977), 369-382.

[4] Gudder, S. P. and Hudson, R. L., A noncommutative propability theory, Trans. Amer. Math. Soc., 245 (1978), 1-41.

[ 5 ] Haagerup, U., Normal weights on $W^{*}$-algebras, J. Functional Anal., 19 (1975), 305-317.

[6] Ikeda, I. and Inoue, A., Invariant subspaces for closed *-representations of *-algebras, Proc. Amer. Math. Soc., 116 (1992), 737-745.

[7] Inoue, A., An unbounded generalization of the Tomita-Takesaki theory, Publ. RIMS, Kyoto Univ., 22 (1986), 725-765.

[8] An unbounded generalization of the Tomita-Takesaki theory II, Publ. RIMS, Kyoto Univ., 23 (1987), 673-726.

[9] Modular structure of algebras of unbounded operators, Math. Proc. Camb. Phil. Soc., 111 (1992), 369-386.

[10] , Modular systems induced by trace functionals on algebras of unbounded operators, J. Math. Phys., 35 (1994), 435-442.

[11] Inoue, A., Kurose, H. and Ôta, S., Extensions of unbounded representations, Math. Nachr., 155 (1992), 257-268.

[12] Katavolos, K. and Koch, I., Extension of Tomita-Takesaki theory to the unbounded algebra of the canonical commutation relation, Rep. Math. Phys., 16 (1979), 335-352.

[13] Lassner, G., Topological algebras of operators, Rep. Math. Phys, 3 (1972), 279-293.

[14] Alegbras of unbounded operators and quantum dynamics, Phisica, 124 A (1984), 471-480.

[15] Powers, R. T., Self-adjoint algebras of unbounded operators, Commun. Math. Phys., 21 (1971), 
85-124.

[16] Powers, R. T., Algebras of unbounded operators, Proc. Sym. Pure Math., 38 (1982), 389-406.

[17] Rieffel, M. A. and Van Daele, A., A bounded operator approach to Tomita-Takesaki theory, Pacific J. Math., 69 (1977), 187-221.

[18] Schmüdgn, K., The order structure of topological *-algebras of unbounded operators I, Rep. Math. Phys., 7 (1975), 215-227.

[19] Unbounded operator Algebras and Representation Theory, Akademie-Verlag Berlin, 1990.

[20] Stratila, S. and Zsido, L., Lectures on von Neumann algebras, Abacus Press, Tunbridge Wells, 1979.

[21] Stratila, S., Modular Theory in Operator Algebras, Abacus Press, Tunbridge Wells, 1981.

[22] Takesaki, M., Tomita's theory of modular Hilbert algebras and its applications, Lecture Notes in Mathematics, Springer, 128 (1970). 
